NASA Contractor Report 195035

ICASE Report No. 95-7
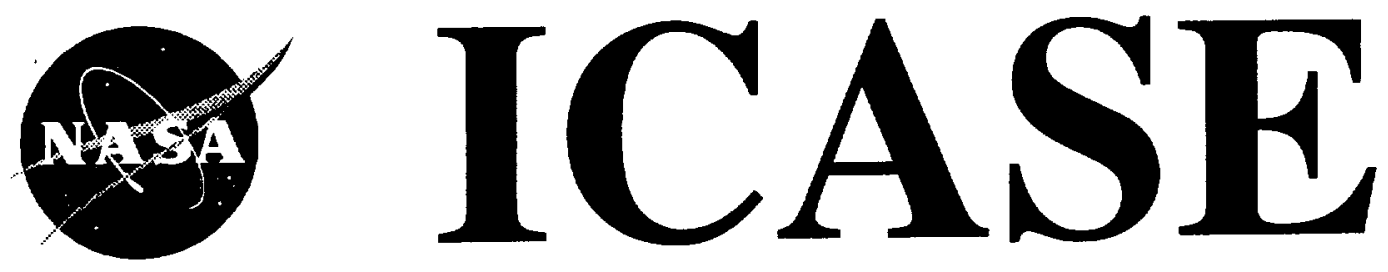

\title{
A WELL-POSED NUMERICAL METHOD TO TRACK ISOLATED CONFORMAL MAP SINGULARITIES IN HELE-SHAW FLOW
}

\section{Gregory Baker \\ Michael Siegel Saleh Tanveer}

(NASA-CR-195035) A WELL-POSEO

NUMERICAL METHOD TC TRACK ISOLATED

CONFORMAL MAP SINGULARITIES IN

HELE-SHAW FLCW Final Report (ICASE) $51 \mathrm{P}$
N95-25114

Unclas

$63 / 640045768$

Contract NAS1-19480

February 1995

Institute for Computer Applications in Science and Engineering NASA Langley Research Center Hampton, VA 23681-0001

SRA Operated by Universities Space Research Association 


\title{
A well-posed numerical method to track isolated conformal map singularities in Hele-Shaw flow
}

\author{
Gregory Baker, Michael Siegel, and Saleh Tanveer* \\ Mathematics Department \\ The Ohio State University \\ Columbus, $\mathrm{OH} 43210$
}

\begin{abstract}
We present a new numerical method for calculating an evolving 2-D Hele-Shaw interface when surface tension effects are neglected. In the case where the flow is directed from the less viscous fluid into the more viscous fluid, the motion of the interface is ill-posed; small deviations in the initial condition will produce significant changes in the ensuing motion. This situation is disastrous for numerical computation, as small round-off errors can quickly lead to large inaccuracies in the computed solution. Our method of computation is most easily formulated using a conformal map from the fluid domain into a unit disk. The method relies on analytically continuing the initial data and equations of motion into the region exterior to the disk, where the evolution problem becomes well-posed. The equations are then numerically solved in the extended domain. The presence of singularities in the conformal map outside of the disk introduces specific structures along the fluid interface. Our method can explicitly track the location of isolated pole and branch point singularities, allowing us to draw connections between the development of interfacial patterns and the motion of singularities as they approach the unit disk. In particular, we are able to relate physical features such as finger shape, side-branch formation, and competition between fingers to the nature and location of the singularities. The usefulness of this method in studying the formation of topological singularities (self-intersections of the interface) is also pointed out.
\end{abstract}

\footnotetext{
* Research was supported by the National Aeronautics and Space Administration under NASA Contract No. NAS1-19480 while the author was in residence at the Institute for Computer Applications in Science and Engineering (ICASE), NASA Langley Research Center, Hampton, VA 23681-0001.
} 



\section{Introduction}

The displacement of a viscous fluid by a less viscous fluid in a Hele-Shaw cell has been the subject of intense investigation over the last decade, mainly due to newly discovered mathematical analogies with dendritic crystal growth, directional solidification, and electro-chemical growth. The original motivation behind the pioneering work of Saffman \& Taylor [36] was the analogy to displacement in porous medium. Reviews by Saffman [38], Bensimon, Kadanoff, Liang, Shraiman, \& Tang [5], and Homsy [16] summarize the state of affairs as of the mid-eighties, while some of the the more recent developments are reviewed by Pelce [31], Kessler, Koplik, \& Levine [22], Howison [21], and Tanveer [42] from a range of different perspectives.

In this paper, we shall limit our investigations to channel flow, although our numerical method is quite general, and can be applied to other geometries. The advantage of this restriction is that our description can be specific. Moreover, the phenomena displayed in channel flow have been studied extensively, and are representative of other Hele-Shaw flows.

A steadily advancing flat interface in a channel is unstable to perturbations when driven by the less viscous fluid. The perturbations grow into fingers, but the subsequent behavior depends on the relative strength of capillary effects, measured by the dimensionless number $B=\sigma b^{2} /\left(12 \mu V a^{2}\right)$. Here $\sigma$ is the surface tension coefficient, $b$ is the gap width of the cell, $a$ is the channel width, $V$ is the speed of displacement well in front of the interface, and $\mu$ is the viscosity of the more viscous fluid (the viscosity of the less viscous fluid is assumed negligible). Numerical computations by Tryggvasan \& Aref [45, 46], DeGregoria and Schwartz [9], Bensimon [6], and Meiburg and Homsy [27] show competition between fingers resulting in the emergence of a single steady finger, provided $B$ is greater than about $0.0004^{\dagger}$ (but not greater than $B_{c}=0.025$ otherwise the interface is stabilized by capillary effects). For still smaller $B$, DeGregoria and Schwartz $[9,10]$ and Bensimon [6] find that the finger spontaneously splits; this can be induced for higher values of $B$ by introducing perturbations at the tip,

\footnotetext{
'There is a range in $B$, depending on the level of noise in an experiment or numerical calculation, in which a transition occurs between steadily moving fingers and continual unsteady motion. A typical range is $0.0005>B>0.0002$. We have taken 0.0004 as a representative value.
} 
even with small amplitude. Experiments also reveal the emergence of tip splitting and side-branching instabilities (Park \& Homsy [28], Tabeling, Zocchi, \& Libchaber [41]). Bensimon [6] provides numerical evidence supporting heuristic arguments that the size of the perturbation triggering instability decreases with $B$. The computations of Dai and Shelley [8] (in the circular geometry) also show great sensitivity to the level of numerical precision when the surface tension coefficient is small. For small enough $B$, even noise during experiments can be large enough to set off a pattern of continual tip-splitting and finger competition ( Maxworthy [26], Arneodo, Couder, Grasseau, Hakim \& Rabaud [2]). Indeed, when capillary effects are very small, it appears that the pattern is fractal (Maxworthy [26], Kopf-Sill \& Homsy [23], Arneodo et. al. [2]).

Detailed understanding of this unsteady behavior is limited. In particular, the numerical work has not produced a clear understanding of the asymptotic trends as $B \rightarrow 0$. Unfortunately, the inclusion of surface tension in the usual mathematical model makes theoretical studies very difficult. Instead a large body of knowledge has been developed for the initial-value problem when $B=0$. For example, Gustaffson $[13,14]$ has rigorously proved the existence of a solution for finite time starting with analytic initial data. Earlier, Galin [11] and Polubarinova-Kochina [32] considered the mathematically identical problem of the Darcy model for ground water flow and devised analytical techniques to obtain exact solutions for a class of initial conditions. These were apparently well-known in the Russian literature (see Hohlov [15] and Howison [21]). Exact solutions due to Saffman [37], Howison [18, 19, 20] and Shraiman \& Bensimon [39] can be seen as applications of these techniques though these results were obtained without knowledge of the earlier Russian work. Howison [21] summarizes the relation between the different techniques. Within the class of known exact solutions, there are finger patterns that exist for all times and exhibit behavior similar to experimental observation (Patterson [30]). Further, there are solutions (Shraiman \& Bensimon [39]) that exist only for a finite time and culminate in a zero angled cusp at the interface. Howison [19] uses the class of known exact solutions to point out that the initial-value problem is ill-posed; it is possible to choose an initial condition for which the solution exists for all times, whereas there is a neighboring initial condition for which the interface develops a cusp after a finite time. 
In essence, these theoretical results use a conformal map $z(\zeta, t)$ which maps the interior of a unit semi-circle in the $\zeta$ plane to the physical flow domain of a channel (see Figure 1). The location of the free surface at a time $t$ is given by $z(\zeta, t)=$ $x(\theta, t)+i y(\theta, t)$ for $\zeta=\epsilon^{i \theta}$ on the arc of the semi-circle. The equation for the evolution of $(x(\theta, t), y(\theta, t))$ results from the usual application of the kinematic and dynamic conditions at the interface. The symmetry in the problem allows us to reflect the solution about the real axis. In essence, we include a mirror image of the channel so that the interface may be considered periodic. Thus, the conformal map must be analytic inside the unit disk, aside from a logarithmic singularity at $\zeta=0$, but it may have singularities and zeros outside it. These can move towards the boundary of the unit disk, $|\zeta|=1$. In particular, zeros may reach the boundary in finite time, causing a cusp to form on the interface. The origin of ill-posedness, then, is that small perturbations can introduce a zero or a singularity in $z_{\zeta}$ near the unit disk, which subsequently moves towards it and reaches it quickly. Following the work of Richardson [35] and Lacey [25], Tanveer [43] showed that all singularities, no matter what type, will move towards the unit disk, while preserving their type. Indeed, we surmise that round-off error in traditional numerical calculations introduces singularities at some distance from $|\zeta|=1$; these subsequently approach the unit disk and lead to the random pattern of tip-splitting, side-branching and finger competition seen in computations for increasingly small $B$.

Ideally, one would like to understand the Hele-Shaw dynamics for a small non-zero $B$ perturbatively, thus exploiting the simpler $B=0$ case. There are several hurdles in accomplishing this. One is the ill-posedness of the $B=0$ problem in the physical domain $|\zeta| \leq 1$. Another is the fact that information in the $B=0$ problem is not complete. We do not have an exact solution to the general initial value problem, nor do we have a detailed understanding of the motion of singularities. Furthermore, it is not known how to numerically compute solutions to the $B=0$ problem effectively. Conventional numerical simulation in the physical flow domain $|\zeta| \leq 1$ suffers from uncontrolled growth of round-off errors (see Aitchison \& Howison [1]). Employing a filtering procedure such as that used by Krasny [24] in the Kelvin-Helmholtz instability (another ill-posed problem) allows simulations in the physical domain to proceed. Still, as is demonstrated by Dai \& Shelley [8], the choice of parameterization has a strong 
effect on the accuracy of the computation. Moreover, a good parameterization is very dependent upon the initial data. For data with a general collection of zeros and singularities, reliable long time calculations in the physical domain become extremely difficult.

In a recent development, Tanveer [43] has been able to demonstrate that analytically extending the initial value problem for $z(\zeta, t)$ into the region exterior to the unit disk leads to a well-posed evolution problem. It is important to note that when the initial interface location is known only to a finite precision, initial data $z(\zeta, 0)$ in the extended region $|\zeta|>1$ cannot be obtained in a well-posed manner. In effect, the ill-posedness of the dynamics is transfered to the ill-posedness of extending the data from $|\zeta|=1$ to $|\zeta|>1$. Nevertheless, when data is specified in $|\zeta|>1$ (say, with $z(\zeta, 0)$ given in closed form) the interface evolves without sensitivity to initial conditions.

Tanveer's observation addresses the first hurdle mentioned above. In this paper, we address the second hurdle by presenting a numerical method which efficiently solves the initial value problem in the expanded domain when $B=0$. Our work extends the method, developed by Baker \& Tanveer [4], to include the trajectories of singularities of the form

$$
z_{\zeta} \sim A(t)\left(\zeta-\zeta_{s}(t)\right)^{\alpha}, \quad \alpha \neq 0,1,2, \ldots
$$

explicitly in the complex plane $\zeta$ when $B=0$. Thus we are able to assess directly the impact of the close approach of pole and branch point singularities to $|\zeta|=1$. We do find that singularities induce tip-splitting and side-branching, and that the relative strength of the singularities control finger competition. Moreover, our studies draw connections between the parameters specifying the singularities and the resulting physical behavior. In other words, for given initial data we are able to predict the outcome from knowledge about the initial singularities in $|\zeta|>1$. Within this framework, comparisons with experimently observed features are possible by studying a random ensemble of initial conditions in $|\zeta|>1$, subject to the constraint that they describe the same initial interface, up to some 'experimental' error.

Beside the explicit treatment of singularities, there is another crucial ingredient in our method. An analytic function, such as a conformal map, is determined completely by its values on a closed curve. Instead of advancing the conformal map on $|\zeta|=1$ in 
time as in standard boundary integral methods, our method advances the conformal map on a much larger circle. The interpolation of the conformal map to $|\zeta|=1$ is then a well-posed operation. In essence, knowledge of the conformal map on a much larger circle corresponds to knowledge of the conformal map on $\left|\zeta^{*}\right|=1$ to a higher degree of precision. In contrast, extrapolation of the conformal map to a larger circle is an ill-posed operation, an operation not to be attempted numerically. Since our method uses the discrete Fourier transform to evaluate Laurent series, our results are spectrally accurate and can be performed in $O(N \log N)$ operations. Furthermore, explicit treatment of the nearest singularities helps avoid point crowding typical of conformal maps.

There are several limitations to our method. First, there is the matter of the initial data. We require that all singularities of $z_{\zeta}$ in the extended domain be isolated branch points or poles. This rules out some types of initial data; for example, structures such as natural boundaries or essential singularities are not allowed in the extended domain. Nevertheless, the kinds of data we do allow produce a wide range of interfacial features such as tip-splitting, side-branching and finger competition which are similar to experimental observations.

The second limitation is that, at present, our method is restricted to the zero surface tension problem. Given this, it is important to discuss the relationship between $B=0$ solutions and those for $B>0$. The well-posed formulation of the $B=0$ problem allows the effects of small surface tension to be addressed perturbatively. It turns out that, at the initial time, surface tension is a regular perturbation except in the neighborhood of zeros and certain singularities of $z_{\zeta}$. Tanveer [43] has examined the effect of small surface tension on isolated singularities in the initial data of the form (1).$^{\ddagger}$ Capillary effects on singularities with $\alpha \leq-4 / 3$ cause only a regular perturbation. Their powers are unchanged but their speed and strengths $A(t)$ are modified slightly by terms proportional to $B$. Singularities with $-4 / 3<\alpha<-1 / 2$ are immediately transformed into a localized cluster of $-4 / 3$ branch point singularities, though the behavior (1) is still relevant in an outer asymptotic sense. Thus, for times much less than $1 / B$ the interface behaves as though it is unaffected by surface tension,

\footnotetext{
tOur use of $\alpha$ for the power corresponds to Tanveer's [43] choice of $-\beta$.
} 
provided these singularities do not come within a distance $O\left(B^{\frac{1}{2+\alpha}}\right)$ from $|\zeta|=1$ and zeros in $z_{\zeta}$ are far from the unit disk. ${ }^{\S}$ For initial conditions with zeros present, recent evidence (Siegel, Tanveer, \& Dai [40]) shows that small surface tension effects can significantly perturb the interface in $O(1)$ time. This can happen even when the zeros do not impinge on the unit disk. The surface tension effects occur in predictable ways when a localized cluster of $-4 / 3$ singularities created out of an initial zero (termed daughter singularity by Tanveer [43]) come within an $O\left(B^{1 / 3}\right)$ neighborhood of the unit disk. However, there are initial conditions for which daughter singularity effects do not occur in $O(1)$ time. Our computations determine the leading order interfacial shapes in such cases. Perhaps more importantly, the method presented here provides a means to determine if and when the zero surface tension evolution of the interface deviates from the small surface tension evolution.

In the next section, we describe the equations upon which our method is based. The explicit treatment of singularities is discussed in Section 3. Then we describe the numerical method in Section 4. In Section 5, we present tests of our method, and some typical results. Our conclusions are discussed in Section 6.

\section{The Equations of Motion}

In this section we present the equations which govern interfacial flow in a Hele-Shaw cell without the details of their derivation. We will follow closely the formulation in Tanveer [43]. Our discussion will be limited to flow in the channel geometry. For the equivalent formulation in a radial geometry, see Tanveer [43].

Consider a Hele-Shaw cell of infinite length and finite width $a$ in which air of negligible viscosity is pushing a viscous, incompressible liquid. Introduce the conformal map $z(\zeta, t)$ which takes the interior of a unit semi-circle in the $\zeta$ plane into the viscous fluid region of the channel, which lies in the $z$ plane. The circular arc $|\zeta|=1$ is mapped to the interface, and the diameter is mapped to the channel walls. A schematic of the mapping is provided in Figure 1. Note that we set the width $a=2$.

\footnotetext{
${ }^{5}$ When singularities are initially $O(1)$ distance from the unit disk, they can move to within $O\left(B^{\frac{1}{2+\alpha}}\right)$ in $O\left(\ln B^{-1}\right)$ time.
} 
The functional form of the conformal map is given by

$$
z(\zeta, t)=-\frac{2}{\pi} \ln \zeta+i+f(\zeta, t)
$$

where $f$ is analytic in an open set which contains the unit semi-circle. The analyticity of $f$ on the circular arc guarantees the smoothness of the interface. We require that

$$
\operatorname{Im}\{f\}=0
$$

on the real diameter of the semi-circle to satisfy the condition that $z$ maps this diameter to the channel walls. In addition, we assume that $z_{\zeta} \neq 0$ in a region containing the unit semi-circle. The Schwartz reflection principle then implies that $f$ is analytic and $z_{\zeta} \neq 0$ for $|\zeta| \leq 1$.

The fluid velocity $\mathbf{u}$, averaged across the plate gap, satisfies Darcy's law

$$
\mathbf{u}=\frac{-b^{2}}{12 \mu} \nabla p
$$

where $\mu$ is the viscosity, $b$ is the plate gap, and $p$ is the pressure (here considered as a function of $x$ and $y)$. Thus $\left(-b^{2} / 12 \mu\right) p$ provides a velocity potential $\phi$. Incompressibility implies the existence of a stream function $\psi$. We can therefore introduce a complex potential function $W(z, t)=\phi(z, t)+i \psi(z, t)$ which is an analytic function of $z$ in the fluid region of the channel. Considered as a function of $\zeta$, this potential is decomposed as

$$
W(\zeta, t)=-\frac{2}{\pi} \ln \zeta+i+\omega(\zeta, t)
$$

where $\omega$ is assumed to be analytic in unit semi-circle, implying (via the Schwartz reflection principle) its analyticity for $|\zeta| \leq 1$. In (4) the velocity at infinity is assumed to be 1 ; together with $a=2$, this choice makes our variables effectively dimensionless. The relation

$$
\operatorname{Im}\{\omega\}=0
$$

is required to hold on the real diameter of $S$, and is the mathematical statement of the condition that there is no flow through the walls.

The interfacial conditions will determine the evolution of the map $z(\zeta, t)$. The kinematic condition states that the normal component of the fluid velocity is continuous 
across the interface and implies that on $|\zeta|=1$

$$
\operatorname{Re}\left\{\frac{z_{t}}{\zeta z_{\zeta}}-\frac{\zeta \boldsymbol{W}_{\zeta}}{\left|z_{\zeta}\right|^{2}}\right\}=0
$$

Details of the derivation of this equation are available in Saffman [37] and Richardson [35]. In the absence of capillary effects, the dynamic condition requires continuity of pressure across the interface. When the viscosity of the less viscous fluid is negligible, this condition gives

$$
\operatorname{Re}\{\omega\}=0
$$

on $|\dot{\zeta}|=1$. We note in passing that a more complete description of Hele-Shaw flow introduces more complicated interfacial conditions due to a thin film left behind by an advancing interface. However, several numerical (Park \& Homsy [29], Reinelt [33, 34]) and analytic (Tanveer [44]) studies have shown that many salient features of interfacial motion described by the more detailed model are captured by the simpler interfacial conditions $(6-7)$.

From (5) and (7) it is immediately apparent that $\omega=0$ for all $\zeta$ in the complex plane. Thus, (6) becomes

$$
\operatorname{Re}\left\{\frac{z_{t}}{\zeta z_{\zeta}}\right\}=-\frac{2}{\pi\left|z_{\zeta}\right|^{2}}
$$

In order to extend (8) into the region $|\zeta|>1$, we first provide an analytic extension of the conjugate of an analytic function evaluated on $|\zeta|=1$. Let

$$
F(\zeta)=\sum_{n=0}^{\infty} a_{n} \zeta^{n}
$$

be an analytic function in $|\zeta| \leq 1$. Then

$$
\tilde{F}(\zeta)=\sum_{n=0}^{\infty} \bar{a}_{n} \zeta^{n}
$$

is analytic in $|\zeta| \leq 1$, where the overbar denotes complex conjugation. For functions that are not necessarily analytic at the origin, though analytic on a segment of $|\zeta|=1$, we can generalize the above definition of $\tilde{F}(\zeta)$ through the relation $\tilde{F}(\zeta)=\bar{F}(\bar{\zeta})$. $\tilde{F}(1 / \zeta)$ is then the analytic extension of $\bar{F}$ from $|\zeta|=1$. It follows that

$$
\operatorname{Re}\{F(\zeta)\}=\frac{1}{2}[F(\zeta)+\tilde{F}(1 / \zeta)]
$$


for $\zeta=\epsilon^{i \theta}$. In addition, $|F(\zeta)|^{2}=F(\zeta) \tilde{F}(1 / \zeta)$ on $\zeta=\epsilon^{i \theta}$. Thus $(8)$ can be written as

$$
\operatorname{Re}\left\{\frac{z_{t}}{\zeta z_{\zeta}}\right\}=-\frac{2}{\pi z_{\zeta}(\dot{\zeta}, t) \tilde{z}_{\zeta}(1 / \zeta, t)}
$$

on $\zeta=\epsilon^{i \theta}$.

The continuation of equation (11) into the domain $|\zeta|<1$ can now be obtained in a straight forward manner by employing the Poisson Integral Formula. In particular, we use a variant of the standard formula which gives the value of an analytic function in the domain $|\zeta| \leq 1$ in terms of its real part evaluated on the unit circle. Application of the formula to the function $z_{t} /\left(\zeta z_{\zeta}\right)$ yields (with appropriate choice of imaginary constant)

$$
\frac{z_{t}}{\zeta z_{\zeta}}=I(\zeta, t)
$$

for $|\zeta|<1$, where

$$
I(\zeta, t)=-\frac{1}{\pi^{2} i} \oint_{\left|\zeta^{\prime}\right|=1} \frac{1}{z_{\zeta}\left(\zeta^{\prime}, t\right) \tilde{z}_{\zeta}\left(1 / \zeta^{\prime}, t\right)} \frac{\zeta^{\prime}+\zeta}{\zeta^{\prime}-\zeta} \frac{d \zeta^{\prime}}{\zeta^{\prime}}
$$

Equation (12) can be analytically continued into the domain $|\zeta|>1$ by deforming the contour in the usual way, producing an additional term from the residue of the pole at $\zeta$. Consequently, we have

$$
z_{t}=\zeta z_{\zeta} I(\zeta, t)-\frac{2 \zeta}{\pi \tilde{z}_{\zeta}(1 / \zeta, t)}
$$

for $|\zeta|>1$. Note that $I(\zeta, t)$ defines different analytic functions in $|\zeta|<1$ and $|\zeta|>1$.

A useful alternative form for (14) may be obtained by employing (10) to write (8) as

$$
\frac{z_{t}}{\zeta z_{\zeta}}=-\frac{\zeta \tilde{z}_{t}(1 / \zeta, t)}{\tilde{z}_{\zeta}(1 / \zeta, t)}-\frac{2 \zeta}{\tilde{z}_{\zeta}(1 / \zeta)}
$$

Since $\tilde{z}_{t}(1 / \zeta)$ and $\tilde{z}_{\zeta}(1 / \zeta)$ are analytic in $|\zeta|>1$, this equation provides us with a second expression for $I(\zeta, t)$.

$$
I(\zeta, t)=-\frac{\zeta \tilde{z}_{t}(1 / \zeta, t)}{\tilde{z}_{\zeta}(1 / \zeta, t)}
$$

when $|\dot{\zeta}|>1$.

In order to make the structure of (12) and (14) more apparent, define

$$
\begin{aligned}
& q_{1}=\dot{\zeta} I(\zeta, t) \\
& q_{2}=\frac{-2 \zeta}{\tilde{z}_{\zeta}(1 / \zeta)} .
\end{aligned}
$$


Then (12) becomes

$$
z_{t}-q_{1} z_{\zeta}=0
$$

for $|\zeta|<1$, and $(14)$ becomes

$$
z_{t}-q_{1} z_{\zeta}=q_{2}
$$

for $|\zeta|>1$. These equations, corresponding to equations (3.1) and (3.3) of Tanveer [43], have the advantage over (8) of allowing studies of the presence and influence of singularities of $a_{\zeta}$ in $|\zeta|>1$. Furthermore, they lead to the development of a new numerical procedure for calculating interfacial motion in a well-posed fashion for a restricted class of initial conditions.

Since $I(\zeta, t)$ and $\tilde{z}_{\zeta}(1 / \zeta)$ are analytic in $|\zeta|>1$, so too are $q_{1}(\zeta, t)$ and $q_{2}(\zeta, t)$, except at infinity, where $q_{1}$ has a simple pole, i.e., grows as $\zeta$. Since $(20)$ has a form analogous to a first order hyperbolic system in the complex plane, (although in reality it is a nonlinear integro-differential equation with coefficients $q_{1}$ and $q_{2}$ that depend nonlocally on $z$ ), the analyticity of $q_{1}$ and $q_{2}$ has important consequences on the presence and motion of singularities of $z$. For the convenience of the reader, we provide a summary of what is known (Richardson [35], Lacey [25], Tanveer [43]):

1. There is no spontaneous generation of singularities in the finite complex plane. Furthermore, the form of a singularity which is present initially in the region $|\zeta|>1$ is invariant with time. Singularities which are present initially at infinity do not move to a finite $\zeta$ location.

2. We define a "characteristic" in the $\zeta$ plane by

$$
\frac{d \zeta_{c}(t)}{d t}=-q_{1}\left(\zeta_{c}(t), t\right)
$$

Let $\zeta_{c}(t)=R_{c}(t) e^{i \theta_{c}(t)}$, then

$$
\frac{\dot{R}_{c}}{R_{c}}=\operatorname{Re}\left\{\frac{\dot{\zeta}_{c}(t)}{\zeta_{c}(t)}\right\}=-\operatorname{Re}\left\{\frac{q_{1}\left(\zeta_{c}(t), t\right)}{\dot{\zeta}_{c}(t)}\right\}
$$

Tanveer [43] has shown that the right hand side of $(22)$ is less than zero when $\left|\zeta_{c}\right|>1$. Consequently, "information" outside of the unit disc flows inward toward $|\zeta|=1$. 
3. This is particularly true for isolated singularities of the form (1). The location of these singularities satisfies $(21)$, i.e. they move with speed $-q_{1}\left(\zeta_{s}(t), t\right)$, so they move towards the unit circle. Incidentally, the singularity in $z$ which is present initially at $\zeta=0$ does not move in time, since the characteristic speed at $\zeta=0$ (given by $q_{1}(0, t)$ ) is zero.

4. Singularities with $\alpha>-1 / 2$ reach the unit circle in finite time. Singularities with $\alpha \leq-1 / 2$ come indefinitely close to, but never reach the boundary $|\zeta|=1$.

Several properties of zeros in $z_{\zeta}$ are also relevant. For example, there is no spontaneous generation of zeros of $z_{\zeta}$ in the finite complex plane, although zeros which are present initially at infinity can move to a finite $\zeta$ location. When a zero impinges on $|\zeta|=1$, it produces a zero-angled cusp in the shape of the interface; see Howison [19. 20] and Shraiman \& Bensimon [39] for exact solutions where this happens. There is no physically sensible way of continuing the solution in time. Unlike singularities, the zeros of $z_{\zeta}$ do not move generally with the characteristic speed $-q_{1}$ and it is hard to predict if a given zero will hit the unit circle or stall at a finite distance from $|\zeta|=1$.

In this paper, our interest will be focussed on initial conditions containing only isolated singularities of form (1) with $\alpha<-1 / 2$, so they do not reach $|\xi|=1$ in finite time. We shall also pick cases where zeros in $z_{\zeta}$ do not reach $|\zeta|=1$ during the time of our computations. For these cases, the inclusion of surface tension acts in a regular perturbative manner, so the results indicate what can be expected in the limit of weak surface tension.

\section{Explicit Treatment of Singularities}

Baker \& Tanveer [4] use $(20)$ to solve directly for $z(\zeta, t)$ on a circle in the $\zeta$ plane of radius $R(t)$, assuming that this circle does not contain a zero of $z_{\zeta}$ or a singularity of $f(\zeta, t)=z-i+(2 / \pi) \ln \zeta$. The advantage of computing the evolution of $f(\zeta, t)$ on the boundary $|\zeta|=R(t)$ is that during the process no singularities in $z_{\zeta}$ will be introduced in $|\zeta| \leq R(t)$. Of course, singularities in $z_{\zeta}$ may be present outside $|\zeta|=R(t)$, but as long as $R(t)$ shrinks fast enough, singularities will not intrude into $|\zeta| \leq R(t)$ : the presence of zeros must be checked separately. Furthermore, a function which is analytic 
in $|\zeta| \leq R(t)$ can be determined throughout this region from its values on $|\zeta|=R(t)$ through evaluation of its Taylor series - this step is well-posed as demonstrated in the next section. 'Thus, the interface, $z(|\zeta|=1, t)$, can be recovered from the solution on $|\zeta|=R(t)$.

Unfortunately, $R(t)$ must shrink faster than the approach of any singularity to $|\zeta|=1$. Consequently, the computation may terminate (when $R=1$ ) before any interesting structures have developed on the interface. For initial conditions containing isolated singularities of form (1), this obstacle may be removed by explicit treatment of the singularities. In this section, we derive equations in which singularities in $|\zeta|>1$ are treated explicitly; they are in a sense 'subtracted' from the data and evolved separately. In the next section, we show how the resulting equations can be solved in an efficient and well-posed fashion.

For initial data containing singularities of form (1) in $|\zeta|>1$, the solution may be written in the form,

$$
\begin{aligned}
z(\zeta, t) & =\sum_{j=1}^{J_{1}} E^{j}(\zeta, t)\left(1-\frac{\zeta}{\zeta_{j}(t)}\right)^{\alpha_{j}+1}+\sum_{j=J_{1}+1}^{J}\left[E^{j}(\zeta, t)\left(1-\frac{\zeta}{\zeta_{j}(t)}\right)^{\alpha_{j}+1}\right. \\
& \left.+\bar{E}^{j}(\zeta, t)\left(1-\frac{\zeta}{\bar{\zeta}_{j}(t)}\right)^{\alpha_{j}+1}\right]+G(\zeta, t)-\frac{2}{\pi} \ln \zeta
\end{aligned}
$$

where $\left|\zeta_{j}\right|>1 ; \alpha_{j}$ are real constants (excluding $\left.0,1,2, \ldots\right)$; and $E^{j}\left(\zeta_{j}(0), 0\right) \neq 0$. Here $\zeta_{j}(t)$ for $j=1, \ldots, J_{1}$ mark the location of singularities on the real line, and $\zeta_{j}(t)$ for $j=J_{1}+1, \ldots . J$ indicate singularities with non-zero imaginary part. In order to satisfy $(3)$, the amplitudes $E^{j}(\zeta, t)$ for $j=1, \ldots, J_{1}$ must be real. The amplitudes $E^{j}(\zeta, t)$ for $j=J_{1}+1 \ldots, J$ are, in general, complex. Conjugate singularities $\bar{\zeta}_{j}(t)$ with amplitudes $\tilde{E}^{j}(\zeta, t)$ are included to satisfy condition $(3)$. The branch cut is chosen so that the argument of $\left(1-\zeta / \zeta_{j}\right)$ lies between $\pi$ and $-\pi$ : we have chosen this form to simplify the numerical evaluation of the interfacial location when $|\zeta|=1$. In the region $|\zeta|>1$, there can be other singularities at $\tilde{\zeta}_{j}$ which are not explicitly represented. However, it is assumed that the $\zeta_{j}$ are the nearest singularities so that, given $i$, the inequality $\left|\tilde{\zeta}_{i}\right|>R(t)>\left|\dot{\zeta}_{j}\right|$ holds for all $j=1, \ldots . J$. The functions $E^{j}(\zeta, t)$ and $G(\zeta, t)$ are analytic in the annulus $1 \leq|\zeta| \leq R(t)$. If all singularities are explicitly represented, these functions are analytic in the entire region exterior to the 
unit disk. Unfortunately, it is possible for $E^{j}$ and $G$ to have singularities in $|\zeta|<1$ which cancel in (23) so that $z+(2 / \pi) \ln \zeta$ is analytic in $|\zeta| \leq 1$.

In order for (23) to be a solution to $(20), \zeta_{j}, E^{j}$, and $G$ must satisfy

$$
\begin{gathered}
\frac{d \zeta_{j}}{d t}=-q_{1}\left(\zeta_{j}(t), t\right) \\
E^{j}{ }_{t}-q_{1} E^{j}{ }_{\zeta}=\left(\alpha_{j}+1\right) E^{j}\left\{\frac{q_{1}(\zeta, t)-q_{1}\left(\zeta_{j}(t), t\right)}{\zeta-\zeta_{j}(t)}-\frac{q_{1}\left(\zeta_{j}, t\right)}{\zeta_{j}(t)}\right\} \\
G_{t}-q_{1} G_{\zeta}=-\frac{2}{\pi \zeta} q_{1}+q_{2} .
\end{gathered}
$$

In the case of initial data with logarithmic singularities (poles in $z_{\zeta}$ ) the solution has a modified form,

$$
\begin{aligned}
z(\zeta, t) & =\sum_{j=1}^{J_{1}} E^{j}(\zeta, t) \ln \left(1-\frac{\zeta}{\zeta(t)}\right)+\sum_{j=J_{1}+1}^{J}\left[E^{j}(\zeta, t) \ln \left(1-\frac{\zeta}{\zeta_{j}(t)}\right)\right. \\
& \left.+\bar{E}^{j}(\zeta, t) \ln \left(1-\frac{\zeta}{\bar{\zeta}_{j}(t)}\right)\right]+G(\zeta, t)-\frac{2}{\pi} \ln \zeta
\end{aligned}
$$

where

$$
\begin{gathered}
\frac{d \zeta_{j}}{d t}=-q_{1}\left(\zeta_{j}(t), t\right) \\
E^{j}{ }_{t}-q_{1} E_{\zeta}^{j}=0 \\
G_{t}-q_{1} G_{\zeta}=q_{2}+\sum_{j=1}^{J} E^{j}\left\{\frac{q_{1}(\zeta, t)-q_{1}\left(\zeta_{j}(t), t\right)}{\zeta-\zeta_{j}(t)}-\frac{q_{1}\left(\zeta_{j}, t\right)}{\zeta_{j}(t)}\right\}
\end{gathered}
$$

Of course, it is possible to have a combination of the two forms, (23) and (27).

Our numerical method is based on $(24-26)$ or $(28-30)$ : in the next section, we describe how the solution may be updated in a well-posed fashion.

\section{Numerical Method}

Our numerical treatment is a combination of tracking the positions of the singularities by solving (24) (or (28)) and of advancing $E^{j}(\zeta, t)$ and $G(\zeta, t)$ in time by the method of lines. Despite appearances, it is not necessary to update $E^{j}(\zeta, t)$, and $G(\zeta, t)$ throughout the computational domain, an annulus $1 \leq|\zeta| \leq R(t)$. hnowledge of these functions on the circles $|\zeta|=1$ and $|\zeta|=R(t)$ is sufficient to determine them everywhere inside the annulus. Nevertheless, we must use a special procedure to update $E^{j}(\zeta, t)$ and $G(\zeta, t)$ in a numerically stable way. 
We introduce a decomposition for a function $g(\zeta)$ which is analytic in the annulus by writing it as the sum of a function $g_{+}(\zeta)$ which is analytic inside a circle $|\zeta|=R$ and a function $g_{-}(\zeta)$ which is analytic outside the circle $|\zeta|=1$. The decomposition is made unique by requiring $g_{+}(0)=0$. By considering the Laurent expansion for $g(\zeta)$, we note that

$$
\begin{aligned}
& g_{-}(\zeta)=\sum_{k=-\infty}^{0} \hat{g}_{k} \zeta^{k} \\
& g_{+}(\zeta)=\sum_{k=1}^{\infty} \hat{g}_{k} \zeta^{k}
\end{aligned}
$$

where the Taylor series for $g_{+}(\zeta)$ will have a radius of convergence $R_{+}>R$, and the Laurent series for $g_{-}(\zeta)$ will converge outside $|\zeta|=R_{-}<1$. We call the function $g_{+}$inner analytic and the function $g_{-}$outer analytic.

An important feature of an inner analytic function is that its values inside a closed curve can be determined from the values on the curve in a well-posed fashion. To see this, consider an inner analytic function $g_{+}(\zeta)$ evaluated on a circle of radius $R$. Let $\dot{\zeta}=R \epsilon^{i \theta}$, then

$$
g_{+}(\theta)=\sum_{k=1}^{\infty} \hat{g}_{k} R^{k} e^{i k \theta}
$$

In practice, the coefficients $\hat{g}_{k} R^{k}$ are obtained by the discrete Fourier Transform. Since the sum converges, these coefficients must decay with increasing $k$. There will be a value, $k_{r}$ say, where the coefficients reach the round-off levels of a computer. If sufficient resolution is used with the discrete Fourier Transform, then the coefficients with $k>k_{r}$ will contain round-off levels and not their actual values. This has important consequences when we wish to evaluate $g_{+}$on a different circle $\zeta=r e^{i \theta}$. Then

$$
g_{+}(\theta)=\sum_{k=1}^{\infty}\left(\frac{r}{R}\right)^{k} \hat{g}_{k} R^{k} e^{i k \theta},
$$

so each coefficient $\hat{g}_{k} R^{k}$ is multiplied by $r^{k} / R^{k}$ prior to using the discrete Fourier Transform again to evaluate the sum. If $r>R$, then round-off errors in $\hat{g}_{k} R^{k}$ for $k>k_{r}$ will be significantly amplified, even to the point that all accuracy in $g_{+}\left(\zeta=r \epsilon^{i \theta}\right)$ will be lost. However, if $r \leq R$, round-off errors are decreased. For an outer analytic function the situation is reversed: evaluation on $r \geq R$ (here we will take $R=1$ ) is numerically stable, while on $r<R$ it is not. The exception to our statements is when 
there is a known, finite number of terms in (31) or (32) with amplitudes well above round-off levels. Then the relative errors in the evaluation of $g_{+}$or $g_{-}$on any circle remains small when we use only the finite number of terms.

\subsection{Basic Algorithm}

We decompose both $E^{j}(\zeta, t)$ and $G(\zeta, t)$ into inner and outer analytic parts and use the method of lines to update $E_{+}^{j}, G_{+}$on $|\zeta|=R$, and $E_{-}^{j}, G_{-}$on $|\zeta|=1$. We obtain evolution equations for these quantities by applying the projection operators, $\mathcal{H}_{+} f=f_{+}$and $\mathcal{H}_{-} f=f_{-}$. Thus, from (25) and (26), we have

$$
\begin{aligned}
E_{-t}^{j}=\mathcal{H}_{-} & \left\{R_{1}(\zeta, t)\right\} \\
\equiv H_{-} & \left\{q_{1} E_{\zeta}^{j}+\left(\alpha_{j}+1\right)\left(\frac{q_{1}(\zeta, t)-q_{1}\left(\zeta_{j}(t), t\right)}{\zeta-\zeta_{j}}-\frac{q_{1}\left(\zeta_{j}, t\right)}{\zeta_{j}(t)}\right) E^{j}\right\} \\
G_{-t}=\mathcal{H}_{-} & \left\{q_{1} G_{\zeta}-\frac{2}{\pi \zeta} q_{1}(\zeta, t)+q_{2}(\zeta, t)\right\} .
\end{aligned}
$$

Before giving the results for the other components, we note that $q_{1}$ and $q_{2}$ have very simple decompositions. From (16) and (17):

$$
q_{1}(\zeta, t)=\zeta \hat{q}_{1}(t)+q_{1_{-}}
$$

where $q_{1_{+}}=\zeta \hat{q}_{1}(t)$ contains only one term, whereas (18) shows that $q_{2}$ is outer analytic only,

$$
q_{2}(\zeta, t)=q_{2}
$$

The form of the decompositions (36) and (37) is very beneficial in our design of a well-posed algorithm. For example, we use the facts that the product of $E_{-{ }_{6}}^{j}$ with $q_{1}$ and the product of $E_{-}^{j}$ with $\left[q_{1}(\zeta)-q_{1}\left(\zeta_{k}\right)\right] /\left(\zeta-\zeta_{k}\right)$ result in only outer analytic parts to simplify the $\mathcal{H}_{+}$projection of (25):

$$
\begin{aligned}
E_{+\imath}^{j} & =\mathcal{H}_{+}\left\{R_{2}(\zeta, t)\right\} \\
& \equiv \mathcal{H}_{+}\left\{q_{1} E_{+\zeta}^{j}+\left(\alpha_{j}+1\right)\left(\frac{q_{1}(\zeta, t)-q_{1}\left(\zeta_{j}(t), t\right)}{\zeta-\zeta_{j}}-\frac{q_{1}\left(\zeta_{j}, t\right)}{\zeta_{j}(t)}\right) E_{+}^{j}\right\}
\end{aligned}
$$

The resulting equation for $C_{+}+$simplifies to

$$
G_{+t}=\mathcal{H}_{+}\left\{q_{1} C_{+i}\right\}
$$

when we use the facts that $-2 q_{1} /(\pi \zeta), q_{2}$ and $q_{1} G_{-\zeta}$ are outer analytic. 
The following properties can be easily deduced from (34-35) and (38-39):

1. The evolution of $E_{+}^{j}$ does not depend explicitly on $E_{-}^{j}$. As a result, if $E^{j}(\zeta, 0)$ satisfies $E_{+}^{j}(\zeta, 0)=0$, then $E_{+}^{j}(\zeta, t)=0$ for all $t$. The same result holds for $G+(\zeta, t)$.

2. If $E_{+}^{j}(\zeta, 0)$ contains a finite number of modes in $(32)$ with highest wavenumber $k=M$, then no modes with wavenumber $k>M$ will be generated in $E_{+}^{j}(\zeta, t)$. The same result holds for $G_{+}$.

Similar results hold for the decomposition of $E^{j}$ and $G$ in (29) and (30) for logarithmic singularities.

To apply the method of lines to (34-35) and (38-39), we assume that we know $E_{+}^{j}$ and $G_{+}$at $N$ evenly spaced points on the circle $r=R(t)$, and $E_{-}^{j}$ and $G_{-}$at $N$ evenly spaced points on the unit circle. Actually, it is not necessary to use the same number of points on both circles, but we make that assumption for ease of presentation. First, we describe how to evaluate the right hand side of $(34)$ and $(35)$ on the unit circle. For the moment we assume that $q_{1}$ and $q_{2}$ are known there; we describe their computation in detail in the next sub-section.

We compute the coefficients $c_{k} R^{k}$ in the representation

$$
E_{+}^{j}\left(\zeta=R \epsilon^{i \theta}, t\right)=\sum_{k=1}^{N / 2} c_{k} R^{k} \epsilon^{i k \theta}
$$

by use of the discrete Fourier Transform. The coefficients $c_{k}$ are obtained by a division by $R^{k}$ and then used to evaluate $E_{+}^{j}$ and $E_{+\zeta}^{j}$ on the unit circle by use of the discrete Fourier Transform, where

$$
E_{+\zeta}^{j}(\zeta, t)=\sum_{k=1}^{N / 2} k c_{k} \zeta^{k-1} .
$$

The function $E_{-\zeta}^{j}$ is also determined on $|\xi|=1$ by using the discrete fourier Transform. Then we have all the information needed to evaluate $R_{1}$ in (34) at $N$ evenly spaced points on the unit circle. To execute the projection $\mathcal{H}_{-}$, we use the discrete Fourier Transform to calculate the coefficients in the representation

$$
R_{1}(\zeta, t)=\sum_{k=-N / 2}^{N / 2} \hat{r}_{k}(t) \zeta^{k} .
$$


Then we set to zero all the coefficients with $k \geq 1$. The result is an outer analytic function, which is the forcing term in (34). Balance of the Fourier coefficients of like modes on the left and right hand sides of (34) then yields a set of equations

$$
\frac{d \hat{E}_{k}^{j}}{d t}(t)=\hat{r}_{k}(t)
$$

for the Fourier coefficients $\hat{E}_{k}^{j}(t)(k \leq 0)$ of $E_{-}^{j}$. In an equivalent procedure, we may use $G_{+}$, known on $r=R(t)$, to determine $G_{+\zeta}$, and use $G_{-}$, known on the unit circle, to get $G_{-\zeta}$. Then the forcing term for (35) is computed by executing the projection $\mathcal{H}_{-}$as described above.

From our discussion on the importance of "characteristics" defined by (21), we anticipate that $E_{+}^{j}$ and $G_{+}$must be evaluated on a circle with a radius that is collapsing at rate,

$$
\frac{1}{R} \frac{d R}{d t}=-\max _{|\zeta|=R(t)} \operatorname{Re}\left\{\frac{q_{1}(\zeta, t)}{\zeta}\right\} .
$$

Then, information outside the circle $|\zeta|=R(t)$ will not cross the boundary into the annulus, $1 \leq|\zeta| \leq R(t)$. We include an advection term that accounts for the change in $E_{+}^{j}$ due to the change in $R(t)$ : thus (38) becomes

$$
\frac{d E_{+}^{j}}{d t}=\mathcal{H}_{+}\left\{R_{2}(\zeta, t)+\frac{\zeta}{R} \frac{d R}{d t} E_{+\zeta}^{j}\right\}
$$

A similar term is needed in the equation (39) for $G_{+}$.

We will describe in detail in the next sub-section how $q_{1}$ and $q_{2}$ may be evaluated on $|\zeta|=R(t)$. The quantities $E_{+_{\zeta}}^{j}, G_{+_{\zeta}}$ can be evaluated by spectral techniques as described in (40). The expression contained within brackets on the right hand side of (42) can then be evaluated. The Fourier coefficients of the right hand side of (42) are obtained using the discrete Fourier Transform, and the projection $\mathcal{H}_{+}$is done by zeroing all modes with $k \leq 0$. Equating like Fourier modes then leads to a set of evolution equations for $\hat{E}_{k}^{j}(t)$, the Fourier coefficients of $E_{+}^{j}$, for $k>0$. The same procedure may be used on the equation for $G_{+}$.

Any suitable ODE Solver may be applied to the evolution equations for $\bar{\zeta}_{j}, \hat{E}_{k}^{j}$, and $\hat{G}_{k}$. For the results used in this paper, we use the standard fourth-order Runge-Kutta method with fixed step-size.

Since $z(\zeta, t)+(2 / \pi) \ln \zeta$ has a Taylor series expansion about $\zeta=0$, all the negative terms in the Laurent expansion for $G(\zeta, t)$ must cancel all the negative terms in the 
Laurent expansions of the sums in (23). This will be only approximately true in the discrete calculation, so it can be used as a check for numerical accuracy. Although it is not necessary to compute $G_{-}(\zeta, t)$ to update the interface, we have done so in order to employ this check.

For certain initial conditions, our algorithm is greatly simplified. For example, when $E_{+}^{j}$ has only a few terms in its Taylor series expansion, then it is possible to write down by hand the evolution equations for the Fourier coefficients, $\hat{E}_{k}^{j}$, since the products

$$
\left(q_{1}+\frac{\zeta}{R} \frac{d R}{d t}\right) E_{+_{\zeta}}^{j} \quad \text { and } \quad \frac{q_{1}(\zeta, t)-q_{1}\left(\zeta_{j}, t\right)}{\zeta-\zeta_{j}(t)} E_{+}^{j}
$$

will contribute only a few terms due to the simple decomposition (36) for $q_{1}$. In particular, if $E_{+}^{j}, E_{-}^{j}, G_{+}$, and $G_{-}$are time-dependent constants in $\zeta$, then only $E_{-}^{j}$ need be computed.

\subsection{Computation of $q_{1}$ and $q_{2}$}

Here we provide details of the computation of $q_{1}$ and $q_{2}$ in $|\zeta| \geq 1$ by using (13), (17), and (18). First, we calculate $E_{+}^{j}(\zeta, t), E_{+\zeta}^{j}(\zeta, t)$, and $E_{-\zeta}^{j}(\zeta, t)$ on $|\zeta|=1$ by means of the discrete Fourier Transform as described in the previous subsection. These values are used to compute $1 / z_{\zeta}(\zeta)=p_{00}(\zeta) / D(\zeta)$ on $|\zeta|=1$ where

$$
\begin{aligned}
D(\zeta) & =-\frac{2 p_{00}(\zeta)}{\pi \zeta}-\sum_{j=1}^{J_{1}} p_{j 0}(\zeta)\left[\left(\alpha_{j}+1\right) \frac{E^{j}(\zeta)}{\zeta_{j}}-E_{\zeta}^{j}(\zeta)\left(1-\frac{\zeta}{\zeta_{j}}\right)\right] \\
& -\sum_{j=J_{1}+1}^{J} p_{0 j}(\zeta)\left[\left(\alpha_{j}+1\right) \frac{\bar{E}^{j}(\zeta)}{\bar{\zeta}_{j}}-\bar{E}_{\zeta}^{j}(\zeta)\left(1-\frac{\zeta}{\bar{\zeta}_{j}}\right)\right] \\
& +G_{\zeta}(\zeta) p_{00}(\zeta)
\end{aligned}
$$

and

$$
p_{r s}(\zeta)=\prod_{\substack{i=1 \\ i \neq r}}^{J_{1}}\left(1-\frac{\zeta}{\zeta_{i}}\right)^{-\alpha_{i}} \prod_{\substack{i=J_{1}+1 \\ i \neq s}}^{J}\left(1-\frac{\zeta}{\bar{\zeta}_{i}}\right)^{-\alpha_{i}}
$$

This form for $1 / z_{\zeta}(\zeta)$ is obtained by differentiating (23) with respect to $\zeta$, then factoring out $1 / p_{00}(\zeta)$ and taking the reciprocal. We have found it necessary to compute $1 / z_{\zeta}(\zeta)$ using this expression to obtain accurate results for $q_{1}$ when singularities are very close to $|\zeta|=1$. The function $1 / \tilde{z}_{\zeta}(1 / \zeta)$ can be computed on $|\zeta|=1$ by taking the conjugate of $1 / z_{\zeta}(\zeta)$. Consequently, the function $q_{2}$ can be computed on the unit circle using (18). 
The function $q_{1}$ is computed as follows. We write

$$
\frac{2}{\pi z_{\zeta}(\zeta) \tilde{z}_{\zeta}(1 / \zeta)}=d_{0}+\sum_{k=1}^{\infty}\left(d_{k} \zeta^{k}+\bar{d}_{k} \zeta^{-k}\right)
$$

on $\zeta=e^{i \theta}$. We determine approximations to the $N$ coefficients $\left\{d_{1}, \ldots, d_{N / 2}\right\}$, $\left\{\bar{d}_{1}, \ldots, \vec{d}_{N / 2}\right\}$ by means of the discrete Fourier Transform. Upon substitution of (44) into (13) and computation of the residues, one finds that

$$
q_{1}=-\zeta\left[d_{0}+2 \sum_{k=1}^{N / 2} \bar{d}_{k} \zeta^{-k}\right]
$$

Note that $d_{j}$ is real (i.e., $\bar{d}_{j}=d_{j}$ ) for the channel geometry, as a consequence of $z$ being real along the real axis of $\zeta$. The functions $q_{1}$ and $q_{2}$ can be analytically continued out to the circle $|\zeta|=R(t)$ by the ideas expressed in (40). Similarly, $q_{1}$ can be evaluated at the location $\zeta_{j}(t)$ of the singularities.

\subsection{Computations for Extremely Close Singularities}

Many of the interesting interfacial features revealed by our calculations occur when singularities approach the unit circle within a distance much less than machine precision. To track these singularities reliably when they are that close to the unit circle we must express their location as

$$
\zeta_{j}=\left(1+\delta_{j}\right) \epsilon^{i \theta_{3}}
$$

The time evolution of the quantities $\theta_{j}(t)$ and $\Delta_{j}(t)=1 / \delta_{j}$ are then determined numerically using the equations

$$
\begin{aligned}
\frac{d \Delta_{j}}{d t} & =\Delta^{2}{ }_{j} \operatorname{Re}\left\{q_{1}\left(\zeta_{j}, t\right) e^{-i \theta_{j}}\right\} \\
\frac{d \theta_{j}}{d t} & =-\frac{1}{1+\delta_{j}} \operatorname{Im}\left\{q_{1}\left(\zeta_{j}, t\right) e^{-i \theta_{j}}\right\} .
\end{aligned}
$$

We use $\Delta_{j}$ instead of $\delta_{j}$ so that singularities can be allowed to come extremely close without the worry of them jumping inside the unit circle due to time-stepping errors.

The computation of $q_{1}\left(\zeta_{j}, t\right)$ is more delicate when $\zeta_{j}$ is extremely close to the unit circle, since the quantities

$$
\frac{1}{\left|\zeta_{j}^{k}\right|}=\frac{1}{\left(1+\delta_{j}\right)^{k}}
$$


in (45) will be approximated as 1 , leading to inaccuracies in the computation. In order to obtain a better value for $1 / \zeta_{j}^{k}$ we write

$$
\frac{1}{1+\delta_{j}}=1-\frac{\delta_{j}}{1+\delta_{j}} \equiv 1+f_{1}\left(\delta_{j}\right)
$$

and compute $f_{k}\left(\delta_{j}\right)$, defined by

$$
\frac{1}{\left(1+\delta_{j}\right)^{k}}=1+f_{k}\left(\delta_{j}\right)
$$

using the recursion

$$
f_{k}\left(\delta_{j}\right)=\frac{f_{k-1}\left(\delta_{j}\right)-\delta_{j}}{1+\delta_{j}}
$$

The substitution of $(46)$ into $(45)$ then yields $q_{1}\left(\zeta_{j}, t\right)=q_{1}\left(\epsilon^{i \theta_{j}}, t\right)+\delta q_{1}\left(\zeta_{j}, t\right)$, where

$$
\delta q_{1}\left(\zeta_{j}, t\right)=-\epsilon^{i \theta},\left(\delta_{j} d_{0}+2 \sum_{k=1}^{N / 2} \bar{d}_{k}\left[\delta_{j}\left(1+f_{k}\right)+f_{k}\right] e^{-i k \theta_{j}}\right)
$$

Then,

$$
\operatorname{Re}\left\{q_{1}\left(\zeta_{j}, t\right) e^{i \theta_{3}}\right\}=\frac{2}{\pi\left|z_{\zeta}\left(e^{i \theta_{3}}, t\right)\right|^{2}} \operatorname{Re}\left\{\frac{\delta q_{1}}{e^{i \theta_{3}}}\right\}
$$

where we have used

$$
\operatorname{Re}\left\{\frac{q_{1}(\zeta, t)}{\zeta}\right\}=\frac{2}{\pi z_{\zeta}(\zeta) \tilde{z}_{\zeta}(1 / \zeta)}
$$

when $|\zeta|=1$, which follows from (13) and (17). By using (48), we avoid any problems with round-off errors. On the other hand, $\operatorname{Im}\left\{q_{1} / \zeta_{j}\right\}$ is computed with $q_{1}$ directly determined from $(4 i)$.

\section{$5 \quad$ Numerical Results}

We use a known exact solution due to Saffman [37] to help validate our method:

$$
z(\zeta, t)=i+d(t)-\frac{2}{\pi} \ln \zeta+\frac{1}{\pi} \ln \left(1-\frac{\zeta^{2}}{a^{2}(t)}\right)
$$

where $1<a(0)<\infty$. The functions $d(t)$ and $a(t)$ are determined by

$$
a(t)=\left[1+K_{1} \epsilon^{-2 \pi t}\right]^{1 / 4}
$$

and

$$
d(t)=K_{0}+2 t+\frac{1}{2 \pi} \ln \left[1+h_{1} \epsilon^{-2 \pi t}\right]
$$


where $K_{0}$ and $K_{1}$ are constants which are determined by the initial conditions. From (49), it is clear that $z_{\zeta}$ has poles at $\zeta_{1,2}= \pm a(t)$, but no zeros. The form of the initial data (27) used in our numerical method is made to correspond to (49) by setting $E^{1}(\zeta, 0)=E^{2}(\zeta, 0)=1 / \pi$ and $G(\zeta, 0)=i+d(0)$. We pick $d(0)$ so that the initial profile has zero mean height. The functions $E^{j}(\zeta, t)$ and $G(\zeta, t)$ are constants in $\zeta$ for this problem, and therefore only a single Fourier mode (namely the constant mode) is necessary to specify them. Nevertheless, we use 512 modes in our computation in order to check the numerical stability of our algorithm for computing $E^{j}(\zeta, t)$ and $G(\zeta, t)$.

The results of our numerical method for $a(0)=2.0$ are shown in Figure 2. We use a time step of $\Delta t=0.005$. At $t=3$, the calculation gives the positions of the poles as $\zeta_{1,2}= \pm 1.0000000244216$, whereas the exact positions are $\zeta_{1,2}= \pm 1.0000000244215$. Although the difference is less than $10^{-12}$, the exact and the numerically computed profiles differ by only a little less than $10^{-6}$. However, we find no growth of round-off errors in the modes comprising $E^{j}(\zeta, t)$ and $G(\zeta, t)$. In this example we set $R(0)=$ 2,000 , and stop the calculation at $t=3$ when $R$ is nearly 1 . Because there are no zeros in $z_{\zeta}$ we can take $R(0)$ much larger and run for even longer times.

There are no known exact solutions for channel geometry when branch point singularities are located in the region $|\zeta|>1$ (although exact solutions with branch point singularities have been found for sector geometry; see Tu [47]). We replace the two pole singularities in $z_{\zeta}$ of (49) by two branch point singularities of equal power and amplitude, and locate them symmetrically on the real axis of $\zeta$. Our choice is motivated by the desire to understand the role of the power of a singularity in $z_{\zeta}$ on the shape of the interface. Thus we select initial data corresponding to (23) with $J_{1}=2, \alpha_{1}=\alpha_{2}$, and $\zeta_{1}(0)=-\zeta_{2}(0)$. In Figure 3, $\alpha_{1}=-4 / 5$, and in Figure 4, $\alpha_{1}=-4 / 3$. Initial values for the singularity positions $\zeta_{j}(0)$ and amplitudes $E^{j}(\zeta, 0)$ are determined through experimentation; those that lead to solutions in which the interface becomes well-deformed before zeros impinge on the unit disc are selected for presentation. In this manner, the initial singularity positions and amplitudes are chosen as $\zeta_{1}(0)=-\zeta_{2}(0)=1.2, E^{1}(\zeta, 0)=E^{2}(\zeta, 0)=1.94$ for Figure 3 , and $\zeta_{1}(0)=-\zeta_{2}(0)=3.3, E^{1}(\zeta, 0)=E^{2}(\zeta, 0)=-2.2$ for Figure 4. In these and all subsequent computations we set $G(\zeta, 0)=i+c$, where $\mathrm{c}$ is a real constant selected to 
produce an initial profile with zero mean height. Recall that for constant initial data such as this, only negative Fourier modes in $E^{j}$ and $G$ are generated. Consequently, we only need to solve equations (34) and (35). The positive Fourier modes are set to zero after each time step in the calculation. We use 512 points on the unit circle in the $\zeta$ plane, and a time step of $\Delta t=0.0005$.

We check our numerical results by comparing them with the results obtained when none of the singularities outside the unit disk are represented explicitly. To perform the latter calculation, we use the code to solve for $G_{+}(\zeta, t)+\hat{G}_{0}(t)$ on a circle of radius $R(t)$ that is closer than the smallest $\left|\zeta_{j}(t)\right|$. Consequently, we shrink the radius $R(t)$ as described in section 4 . While the calculation must be stopped before long because $R \leq 1$, the output provides a useful check on the present results for some period of time. In addition, we decrease the time step and increase the number of modes until there are no detectable differences in the solution within plotting accuracy.

Unlike the previous example, we cannot be sure that zeros do not approach the unit disk. So we monitor the presence of zeros by computing the integral

$$
N_{z}=\frac{1}{2 \pi i} \oint_{|\zeta|=1} \frac{z_{\zeta \zeta}}{z_{\zeta}} d \zeta
$$

which, according to the argument principle, equals the number of zeroes minus the number of poles of $z_{\zeta}$ inside the unit disk. As long as there are no zeros of $z_{\zeta}$ in $|\zeta| \leq 1$, this integral will equal -1 , due to the simple pole of $z_{\zeta}$ at $\zeta=0$. For the results presented in Figures 3 and 4 , the value of $N_{z}$ remains within $1 \%$ of -1 throughout the length of the computation. Note further that if a zero in $z_{\zeta}$ is located near the unit disk, the close presence of a pole singularity in the integrand of (50) causes a loss of accuracy in its numerical evaluation. Thus we feel confident that no zeros are very near to the unit disk up until the times of the final profiles in Figures 3,4 , and 5 .

The contrast between Figures 3 and 4 is quite striking. The higher value of $\alpha=-4 / 5$ in Figure 3 correspond to a singularity of weaker effect in that the bulge of fluid at the base of the finger is less rounded, causing a finger that is more pointed. The lower value of $\alpha=-4 / 3$ in Figure 4 produces a more spherically shaped bulge of fluid, causing a thinner neck at the base of the finger. In general, our experiments with various powers $\alpha$ show that for powers $\alpha>-1$, more pointed fingers are produced 
from the less pronounced bulges of fluid at their bases. For $\alpha<-1$, the fingers have parallel sides, but their bases have thinner necks as a consequence of more spherically rounded bulges of fluid there.

A representative Fourier spectrum from the calculation with $\alpha=-4 / 3$ is presented in Figure 5. There is no sign of spurious growth in the large $|k|$ modes or any other indications of numerical instability, although it is necessary to evaluate $q_{1}$ using expression (43) in order to avoid such growth. The rise in the tail of the spectrum with respect to time is due to singularities in the functions $E^{j}, G, q_{1}$, and $q_{2}$ at positions $\zeta=1 / \zeta_{1}$ and $\zeta=1 / \zeta_{2}$ inside the unit disc. Since $z-(2 / \pi) \ln \zeta$ is analytic in $|\zeta|<1$, the singularities in $E^{j}$ and $G$ must cancel out in the expression for $z(\zeta),(23)$. Nevertheless, these singularities do affect the numerical computation of the quantities $E^{j}, G, q_{1}$, or $q_{2}$. Since the singularities move toward $\zeta= \pm 1$ from inside the unit disc as $\zeta_{1,2} \rightarrow \pm 1$ from the outside, a large number of Fourier modes are required to obtain accurate representations for these quantities when the singularities are very close to the unit circle. Another consequence of the close approach of the singularities to the unit circle is the need for much smaller time steps to maintain accuracy. This is also true when zeros in $z_{\zeta}$ get close to the unit disk. In general, it is either the close approach of singularities in $E^{j}$ and $G$ or zeros in $z_{\zeta}$ to $|\zeta|=1$ that force us to terminate our calculations.

If we ignore the loss of accuracy, we can continue the calculations shown in Figures 3 and 4 a little further in time. We see evidence that zeros are impinging on the unit disk. For the calculations associated with Figure 3, a zero is approaching $|\zeta|=1$ at a point corresponding to the tip of the finger. Thus, we expect the finger to form a cusp in finite time. For Figure 4, two zeros approach $|\zeta|=1$ at points corresponding to the tops of either side of the finger, so we expect cusps to form at these positions. Asymptotic theory suggests that the initial zeros will give rise to localized clusters of $-4 / 3$ singularities (daughter singularities) when $0<B<1$. The leading order motion of each of these clusters satisfies equation (21), i.e., a cluster located at $\zeta_{d}$ moves with speed $-q_{1}\left(\zeta_{d}(t), t\right)$. If such a cluster comes close to $|\zeta|=1$, it can cause the interface to deviate significantly from the $B=0$ solution.

We briefly consider the influence of non-zero surface tension on interfacial shapes by comparing the zero surface tension solution to that for small surface tension. The 
non-zero $B$ solution is obtained using the boundary integral method developed by Hou, Lowengrub \& Shelley [17]. In the first case (Figure 6a), we consider initial data with zeros of $z_{\zeta}$ initially placed at infinity, and with singularities which satisfy $\alpha_{j} \leq-4 / 3 . \pi$ The value of surface tension is set to $B=.0025$. From asymptotic theory, it is expected that the addition of a small amount of surface tension will make little difference in the evolution of the interface for at least $O(\ln B)$ time. The actual agreement between the interfacial shapes is quite remarkable: the two solutions are indistinguishable over the entire length of the run. The agreement is unaffected by using real singularities which only satisfy $\alpha_{j}<-1 / 2$, since these singularities behave as though they are essentially unaffected by capillary effects for the time of the computation.

In contrast, when the initial data of Figure 4 is used, the difference between the $B=0$ and $B=.0025$ shapes is very significant. As seen in Figure $6 \mathrm{~b}$, the $B>0$ finger eventually diverges from the corresponding zero surface tension solution and approaches a broad, steadily propagating finger. The broadening is apparently caused by the approach of daughter singularities created from an initial zero in $|\zeta|>1$ (see Siegel, Tanveer \& Dai [40]).

The above examples show that, in some cases, surface tension causes a singular perturbation in $O(1)$ time, whereas in other cases it does not. Our method gives us a means of discerning these cases through computation of daughter singularity trajectories in the extended domain [43]. We remark that our attempts to calculate the $B=0$ solutions using the boundary integral method with filtering failed before the interface had advanced very far. This is because the close presence of strong singularities causes fast growth of the high wavenumber modes, and numerical noise quickly contaminates the computation. Thus, boundary integral methods often appear unsuitable for comparing $B=0$ solutions to those for $B>0$ over times in which the interface becomes significantly deformed. Dai \& Shelley [8] report related problems in $B=0$ calculations, as discussed in the introduction.

We turn now to a consideration of the influence of additional $-4 / 3$ singularities with weak amplitudes during finger formation. Figure $7 a$ illustrates the interface evolution resulting from initial data of the form (23) with $J_{1}=2, J=3$ and with

\footnotetext{
"This is accomplished by prescribing initial data in $z_{\zeta}$ of the form $z_{\zeta}(\zeta, 0)=-2\left(1-\zeta^{2} / \zeta_{s}^{2}\right)^{\alpha} /(\pi \zeta)$. Here we use $\alpha=-3.2$ and $\zeta_{s}=(1.6,0.0)$.
} 
singularity strengths $\alpha_{j}=-4 / 3$ for $j=1, \ldots, 3$. The initial singularity positions were chosen as $\zeta_{1}(t)=-\zeta_{2}(t)=(1.65,0.0)$, and $\zeta_{3}(t)=(-0.6,1.83)$, and the initial amplitudes as $E^{1}(\zeta, 0)=E^{2}(\zeta, 0)=(-0.9,0.0)$, and $E^{3}(\zeta, 0)=(-0.01,0.03)$. The calculation uses $N=512$ points and a time step $\Delta t=0.0005$. Two of the singularities $\left(\zeta_{1}\right.$ and $\left.\zeta_{2}\right)$ reside on the real line, and these have large enough amplitude to produce the wide bulges of fluid centered at $y=1$ and $y=-1$ which define the main finger. As the third singularity approaches the unit disk, it generates a small bulge of fluid that gives the appearance of the formation of a dimple on the evolving finger.

The dimple is clearly stationary in the laboratory frame, despite the overall growth of the main finger. Such behavior has been well documented in laboratory experiments ( e.g. Park \& Homsy [28], Tabeling, Zocchi, \& Libchaber [41]) and in numerical calculations (e.g. DeGregoria and Schwartz $[9,10]$ ) with $B \neq 0$. Our results show that it is the specific nature of the trajectory of the singularity $\zeta_{3}(t)$ that accounts for this behavior. We show the trajectories of all the singularities in Figure 7b. At first, a dimple starts to form as $\zeta_{3}(t)$ approaches the unit disk. As $\zeta_{3}(t)$ begins to move around the boundary of the unit disk towards $\zeta=-1$, the dimple continues to grows but remains stationary in the laboratory frame. With the assumption that the singularity is close to either $\zeta= \pm 1$, we can show that its speed is actually the correct speed for it to remain stationary in the laboratory frame. Details will be provided elsewhere. The point to be made here is only that certain physical properties can be understood in terms of the motion of singularities in the complex $\zeta$ plane. Under the presence of surface tension, the narrow finger will eventually widen; however, the formation of the dimple and its relationship to the motion of the singularities will not be affected.

Placing additional singularities in the complex plane produces additional dimples and leads to the appearance of side-branching. An example of side-branching due to multiple pole singularities is given in Figure $8 \mathrm{a}$. We use pole singularities, rather than $\alpha=-4 / 3$ singularities, since our ability to track them arbitrarily close to the unit disc leads to a more dramatic example of sidebranching, but in reality side-branching is more likely to occur from the patterns of $-4 / 3$ singularities created by the initial transformation of zeros in $z_{\zeta}$. Initial data corresponding to $(27)$ with $J_{1}=2$ and $J=14$ is used to generate the profiles. The starting amplitudes $E^{j}(\zeta, 0)$ and singularity 
positions $\zeta_{j}(0)$ are given in Table I. The two main singularities $\zeta_{1}$ and $\zeta_{2}$ produce the finger centered in the channel, and remaining singularities $\zeta_{j}$ for $j=3, \ldots, J$ of smaller amplitude are located to cause side-branches to form near the base of the finger. Additional singularities can be placed to allow side-branching to run the length of the finger. As expected, the dimples and the corresponding side-branches are stationary in the laboratory frame. The motion of the singularities is shown in Figure $8 \mathrm{~b}$, and clearly the singularities with smaller amplitudes are attracted to the points $\zeta= \pm 1$. When they get close enough to either of these points, an unperturbed finger continues to grow while the dimples remain near its base. If additional singularities are present that start much further away from the unit disk, they will arrive close to the unit disk at later times, producing new dimples near the tip of the finger. The endless presence of singularities streaming in from infinity can generate fingers with side-branching patterns seen frequently in experiments.

In Figure $7 \mathrm{a}$, the small amplitude of the singularity at $\zeta_{3}$ causes the formation of a small dimple on the side of a well-developed finger. In contrast, when $\operatorname{Re} E^{3}$ is the same order as $\operatorname{Re} E^{1}$ and $\operatorname{Re} E^{2}$, the close approach of $\zeta_{3}$ to $|\zeta|=1$ leads to a 'tip-splitting' event and the formation of two fingers which eventually compete. An example is given in Figure 9a for initial data of the form (23) with $J_{1}=2, J=$ 3 and $\alpha_{j}=-4 / 3$ for $j=1, \ldots, 3$. The initial singularity positions are chosen as $\zeta_{1}(0)=-\zeta_{2}(0)=(2.5,0.0), \zeta_{3}(0)=(0.0,4.0)$ and the initial amplitudes as $E_{1}(\zeta, 0)=$ $(-1.0,0.0), E_{2}(\zeta, 0)=(-0.8,0.0), E_{3}(\zeta, 0)=(-0.3,-0.16)$. We use $N=512$ and $\Delta t=0.0005$. As shown in Figure $9 \mathrm{~b}$, the singularities initially move radially towards the unit circle. As the singularity at $\zeta_{3}$ approaches $|\zeta|=1$, a dimple forms near the finger tip. As time advances, $\zeta_{3}$ gets closer to the interface and the dimple elongates into a large indentation, giving the appearance of two fingers. Eventually, the motion of the singularity is predominantly tangent to the circle, and the tangential velocity of $\zeta_{3}$ is such that the indentation is fixed in the laboratory frame. The direction the singularity moves around the circle determines which finger will dominate. In this case the singularity at $\zeta_{3}$ moves towards $\zeta=-1$. Since the point $\zeta=-1$ corresponds to the bottom end of the interface, this motion has the effect of stretching the interface so that the indentation separating the fingers lies closer to the bottom end. The only way this can be done while also keeping the indentation fixed in the laboratory frame 
is for the upper finger to grow significantly more than the lower one, as indicated in Figure 9a. Unfortunately, the simulation cannot be not run long enough to produce a clear outcome in the finger competition, because of the presence of the singularity in $E_{-}^{j}$ and $G_{-}$at $1 / \zeta_{3}(t)$. Nevertheless, we are able to compute long enough to make the trend in singularity motion clear.

We conclude this section with a brief examination of some scenarios which may lead to a self-intersection of the interface. A self-intersection event is often referred to as a topological singularity; the possible formation of this type of singularity in Hele-Shaw flow and in other free surface flows is a topic of much current interest. A topological singularity occurs when the conformal map $z(\zeta, t)$ ceases to be univalent, i. e., when two points on the $\zeta$ semi-circle map to a single point. When this happens, either the more viscous or less viscous fluid region is divided into two disjoint sections. Bertozzi, Brenner, Dupont, \& Kadanoff [7] and Goldstein, Pesci, \& Shelley [12] have investigated possible topological singularity formation in Hele-Shaw flow with surface tension. They consider a particular geometry, consisting of a vertical cell with a thin layer of fluid resting on the bottom, chosen so that a variant of the lubrication approximation can be applied. Using this approximation, they concluded that in certain cases the top interface of the layer touches the bottom of the cell in finite time. However, their geometry and pressure conditions are significantly different from ours and it is unclear if their results can be extrapolated to our geometry, where there is a constant pressure gradient far ahead of the finger.

In some situations, a loss of univalence is possible even when the singularities and zeroes of $z_{\zeta}(\zeta, t)$ in $|\zeta|>1$ remain a finite distance from $|\zeta|=1$. We used our code to search for such an event in the zero surface tension problem. Unlike commonly used boundary integral methods which run into resolution difficulties when the interface is about to pinch (see Baker \& Shelley [3]), our numerical approach based on the conformal mapping function will not incur difficulties as long as the pinching is not accompanied by a singularity or zero of $z_{\zeta}$ impinging on $|\zeta|=1$.

Unfortunately, we were unable to find any occurance of this type of singularity in the situation where a fluid of zero viscosity displaces a viscous fluid. Of course, the formation of a topological singularity in this case cannot be completely ruled out from our limited examination and further study is required. When we reversed the 
pressure gradient at infinity, however, so that the more viscous fluid on the right of Figure 1 displaces the fluid of negligible viscosity on the left, topological singularities were sometimes observed. It is well known from the Saffman \& Taylor [36] analysis that a planar interface in this situation is stable and that any small deformation will reduce with time. This can be expected to be true even for most finite amplitude disturbances. However, our findings show that if the interface is highly deformed initially, pinching can occur.

One such example is presented in Figure 10. This figure shows interfacial profiles before and after topological singularity formation for an initial value problem with two branch point singularities initially close to the unit disk. We picked data in $z_{\zeta}$ of the form $z_{\zeta}(\zeta, 0)=-2\left(1-\zeta^{2} / \zeta_{s}^{2}\right)^{\alpha} /(\pi \zeta)$ where $\alpha=-1.9$ and $\zeta_{s}=(1.105,0.0)$; with this choice, zeros in $z_{\zeta}$ are placed initially at infinity." The change to liquid pushing air reverses the direction of the characteristic velocities (given by $q_{1}$ ), and the singularities move outward from the unit circle. Consequently, the evolution of the interface can be obtained without explicitly representing the singularities. We therefore set $z-(2 / \pi) \ln \zeta=G_{+}+\hat{G}_{0}$ and solve $(39)$ on the circle $|\zeta|=e^{i \nu}$; there is no need to evolve the radius of this circle. In the run we set $N=128$ and $\Delta t=0.001$.

Our example shows that a loss of univalency can occur in zero surface tension flows without concurrent singularities in $z_{\zeta}$, and illustrates the ability of our numerical method to contend with such self intersections. The occurance of these singularities appears to be quite sensitive to initial conditions, with fatter initial fingers typically evolving to a flat sheet without pinching. The form of the solution after a self-intersection remains an open question.

\section{Discussion and Conclusions}

We have described a numerical method designed to track singularities present in the conformal map from the unit semi-circle to the physical domain of Hele-Shaw flow in a channel. The method is restricted to those conformal maps that contain singularities of the form (23), and their initial location $\zeta_{j}(0)$ and power $\alpha_{j}$ must be given if they lie

\footnotetext{
"Thus, the presence of surface tension will not significantly affect the evolution of the interface for the times shown.
} 
inside the circle of radius $R(0)$. Furthermore, their amplitude $E^{j}(\zeta, 0)$ and $G(\zeta, 0)$ must be specified, or at least their inner and outer components must be given on $|\zeta|=R(0)$ and $|\zeta|=1$ respectively. This detailed information of the initial properties of the conformal map correspond to high precision knowledge of the initial interface location. Our numerical method then advances the conformal map, and hence the interface, numerically in a stable way. In other words, round-off errors do not contaminate the high precision specification of the interface location.

Besides the restrictions on the initial conditions, there are two other limitations on our method. Singularities in inner analytic components of $E^{j}(\zeta, t)$ and $G(\zeta, t)$ occur inside the unit disk at $1 / \zeta_{j}(t)$. As $\zeta_{j}(t)$ approaches the unit disk, these singularities also approach the boundary of the unit disk, and cause slow decay of the Fourier modes for $E^{j}(\zeta, t)$ and $G(\zeta, t)$. Consequently, we need many Fourier modes to ensure reasonable accuracy. Also, at present the method is limited to $B=0$. When $B \neq 0$, zeros in $z_{\zeta}$ are transformed into patterns of $-4 / 3$ singularities. Some of these can move toward the unit circle and affect the shape of the interface at later times. Nevertheless, as long as $\alpha_{j} \leq-4 / 3$ and none of the singularities formed out of initial zeros approach the unit disk closely, our results will be the correct limiting behavior as $B \rightarrow 0$. Perhaps more importantly, our method enables accurate $B=0$ computations to be obtained for quite a general distributions of initial singularities, so that comparisons with $B>0$ solutions can be made. These kind of comparisons complement the asymptotic theory, and facilitate an understanding of the influence of small capillary effects.

Despite the limitations, we find singularities induce interfacial structure that is typical of experiment observations when $B$ is very small. In particular, two singularities, placed on the real axis on either side of the unit circle, induce formation of a long finger. Singularities off the real axis induce small indentations on this finger if their amplitudes are small, giving the appearance of side-branches, or large indentations if their amplitudes are comparable to the ones on the real axis, giving the appearance of tip-splitting and finger competition. In general, we expect that a continual inward stream of singularities of all amplitudes can account for multiple branching and competition as observed experimentally. Although some aspects of interfacial evolution due to multiple singularities have been examined previously by Howison [19] using a 
class of exact solutions for simple pole singularities, our algorithm allows a broader study to be undertaken for collections of isolated singularities of more general form.

We plan to continue studies of the properties of the singularities and the interfacial structure their induce. For example, we wish to explore what role the complex amplitudes have on the trajectories of the singularities. We hope to find ways to represent the singularities in better forms that may remove some of the limitations in our method, and we hope to find ways to capture the transformation of zeros when $B$ is very small, but non-zero. The authors would like to acknowledge support from NASA grant NAG 3-1415 (G.B.), Department of Energy contract DE-FG02-92ER14270 (M. S. and S. T.), and an NSF Postdoctoral Fellowship (M. S.). S. T. was partially supported by NASA grant NAS1-18605 while in residence at The Institute for Computer Applications in Science and Engineering (ICASE). 


\section{References}

[1] Aitchison, J.M. \& Howison, S., Computation of Hele-Shaw flows with free boundaries, J. Comput. Phys., 60, 376 (1985).

[2] Arneodo, A., Couder, Y., Grasseau, C., Hakim, V. \& Rabaud, M., Uncovering the analytical Saffman-Taylor finger in unstable viscous fingering and diffusion limited aggregation, Phys. Rev. Lett., 63, 984 (1989).

[3] Baker, G. \& Shelley, M., Boundary integral techniques for multi-connected domains, J. Comput. Phys., 64, 112 (1986).

[4] Baker, G. \& Tanveer, S., Well-posed numerical calculations for free-surface flows, in Singularities in Fluids, Plasmas and Optics, edited by R. Caflisch (Kluwer, Boston, 1992), p.1.

[5] Bensimon, D., Kadanoff, L.P., Liang, S., Shraiman, B.I., \& Tang, C., Viscous flows in two dimensions, Rev. Mod. Phys., 58, 977 (1986).

[6] Bensimon, D., Stability of viscous fingering, Phys. Rev. A, 33, 1302 (1986).

[7] Bertozzi, A.L., Brenner, M.P., Dupont, T.F., Kadanoff, L.P., Singularities and similarities in interface flows, Trends and Perspectives in Applied Mathematics, Vol. 100, edited by L. Sirovich (Springer, New York, 1994), pp. 155-208.

[8] Dai, W. \& Shelley, M., A numerical study of the effect of surface tension and noise on an expanding Hele-Shaw bubble, to appear in Phys. Fluids A.

[9] DeGregoria, A.J. \& Schwartz, L.W., Finger breakup in Hele-Shaw cells, Phys. Fluids, 28, 2313 (1985).

[10] DeGregoria, A.J. \& Schwartz, L.W., A boundary-integral method for two-phase displacement in Hele-Shaw cells. J. Fluid Mech., 164, 383 (1986).

[11] Galin, L.A., Dokl. Akad. Nauk. S.S.S.R., 47, 246 (1945) (in Russian).

[12] Goldstein, R., Pesci, A., \& Shelley, M., Topology transitions and singularities in viscous flows, Phys. Rev. Lett, 70 (20), 3043 (1984). 
[13] Gustaffson, B., Existence of weak backward solutions to a generalized Hele-Shaw moving boundary problem, Nonlinear Analysis, T.M.A., 9, 203 (1984).

[14] Ciustaffson, B., An ill-posed moving boundary problem for doubly-connected domains, Arkiv Fur Mathematik, 25, 231 (1987).

[15] Hohlov, Y. E., M.I.A.N. Preprint No. 14, Steklov Institute, Moscow.

[16] Homsy, G.M., Viscous Fingering, Ann. Rev. Fluid Mech., 18, 271 (1987).

[17] Hou, T., Lowengrub, J. \& Shelley, M., Removing the stiffness from interfacial flows with surface tension, J. Comp. Phys., 114, 2 (1994) 312-338.

[18] Howison, S.D., Bubble growth in porous media and Hele-Shaw cells, Proc. Roy. Soc. Edin. A, 102, 141 (1985).

[19] Howison, S.D., Fingering in Hele-Shaw cells, J. Fluid Mech., 167, 439 (1986).

[20] Howison, S.D., Cusp development in Hele-Shaw flow with a free surface, SIAM J. Appl. Math, 46, 20 (1986).

[21] Howison, S.D., Complex variable methods in Hele-Shaw moving boundary problems, European J. Appl. Math 3, 209 (1991).

[22] Kessler, D., Koplik, J. \& Levine, H., Pattern selection in fingered growth phenomena, Advances in Physics, 37, 255 (1988).

[23] Kopf-Sill, A. R. \& Homsy, G.M., Nonlinear unstable viscous fingers in Hele-Shaw flows. I. Experiments, Phys. Fluids, 31, 18 (1988).

[24] Krasny, R., A study of singularity formation in a vortex sheet by the point-vortex approximation, J. Fluid Mech, 167, 65 (1986).

[25] Lacey, A.A., Moving boundary problems in the flow of liquid through porous media, J. Australian Math. Soc., B24, 171 (1982).

[26] Maxworthy, T.. The nonlinear growth of a gravitationally unstable interface in a Hele-Shaw cell, J. Fluid Mech., 177, 207 (1987). 
[27] Meiburg, E., \& Homsy, G.M., Nonlinear unstable viscous fingers in Hele-Shaw flows. II. Numerical simulation. Phys. Fluids, 31, 429 (1988).

[28] Park, C.W. \& Homsy, G.M., The instability of long fingers in Hele-Shaw flows, Phys. Fluids, 28, 1583 (1985).

[29] Park, C.W. \& Homsy, G.M., Two-phase displacement in Hele-Shaw cells: Theory., J. Fluid Mech., 139, 291 (1985).

[30] Patterson, L., Radial fingering in a Hele-Shaw cell, J. Fluid Mech, 113, 513 (1981).

[31] Pelce, P. 1988, Dynamics of Curved Fronts, (Academic Press, San Diego).

[32] Polubarinova-Kochina, P., Dokl. Akad. Nauk. S.S.S.R, 47, 254 (1945) (in Russian).

[33] Reinelt, D.A., Interface conditions for two-phase displacement in Hele-Shaw cells, J. Fluid Mech, 183, 219 (1987).

[34] Reinelt, D.A., The effect of thin film variations and transverse curvature on the shape of fingers in a Hele-Shaw cell, Phys. Fluids, 30, 2617 (1987).

[35] Richardson, S., Hele-Shaw flows with a free boundary produced by the injection of fluid into a narrow channel, J. Fluid Mech., 56, 609 (1972).

[36] Saffman, P.G. \& Taylor, G.I., The penetration of a fluid into a porous medium of Hele-Shaw cell containing a more viscous fluid, Proc. $R$. Soc. London Ser. A, 245, 312 (1958).

[37] Saffman, P.G., Exact solution for the growth of fingers from a flat interface between two fluids, Q. J. Mech. appl. Math., 12, 146 (1959).

[38] Saffman, P.G., Viscous fingering in a Hele-Shaw cell. J. Fluid Mech., 173, 73 (1986).

[39] Shraiman, B.I. \& Bensimon, D., Singularities in nonlocal dynamics, Phys. Rev. A, 30, 2840 (1985). 
[40] Siegel, M., 'Tanveer, S. \& Dai, W., Singular Perturbation of a smoothly evolving zero surface tension Hele-Shaw flow-a comparison between asymptotics and numerics, to be submitted to J. Fluid Mechanics.

[41] Tabeling,P, Zocchi, G.\& Libchaber, A., An experimental study of the SaffmanTaylor instability, J. Fluid Mech., 177, 67 (1987).

[42] Tanveer, S., Viscous displacement in a Hele-Shaw cell, in Asymptotics Beyond All Orders, edited by H. Segur, S. Tanveer and H.Levine (Plenum, New York, 1991), p. 131.

[43] Tanveer, S., Evolution of a Hele-Shaw interface for small surface tension, Phil. Trans. R. Soc. London A., 343, 1 (1993) (see also erratum 1994).

[44] Tanveer, S., Asymptotic calculation of thin-film effects on Hele-Shaw fingering, submitted to J. Fluid Mech.

[45] Tryggvasson, G. \& Aref, H., Numerical Experiments on Hele-Shaw flow with a sharp interface, J. Fluid Mech., 136, 1 (1983).

[46] Tryggvason, G. \& Aref, H., Finger-interaction mechanisms in stratified Hele-Shaw flow, J. Fluid Mech., 154, 287 (1985).

[47] Tu, Y., Saffman-Taylor problem in sector geometry, in Asymptotics Beyond All Orders, edited by H. Segur, S. Tanveer and H.Levine (Plenum, New York, 1991), p.175. 

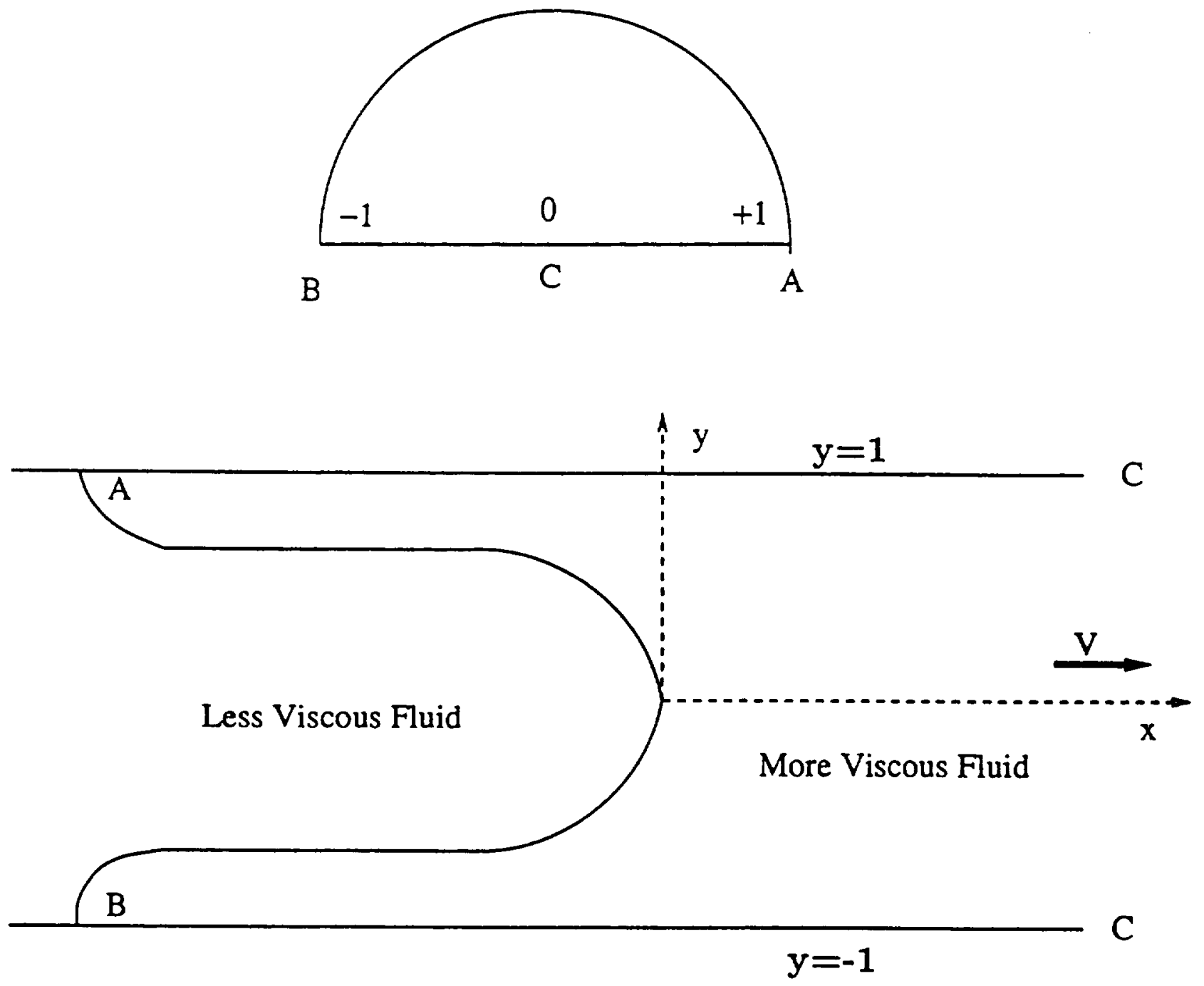

1. The unit semi-circle in the $\zeta$ plane is mapped into the viscous fluid region of the channel, with the circular arc being mapped to the interface. The points $A, B$, and $\mathrm{C}$ in the $\zeta$ plane are mapped to the corresponding points in the channol. 


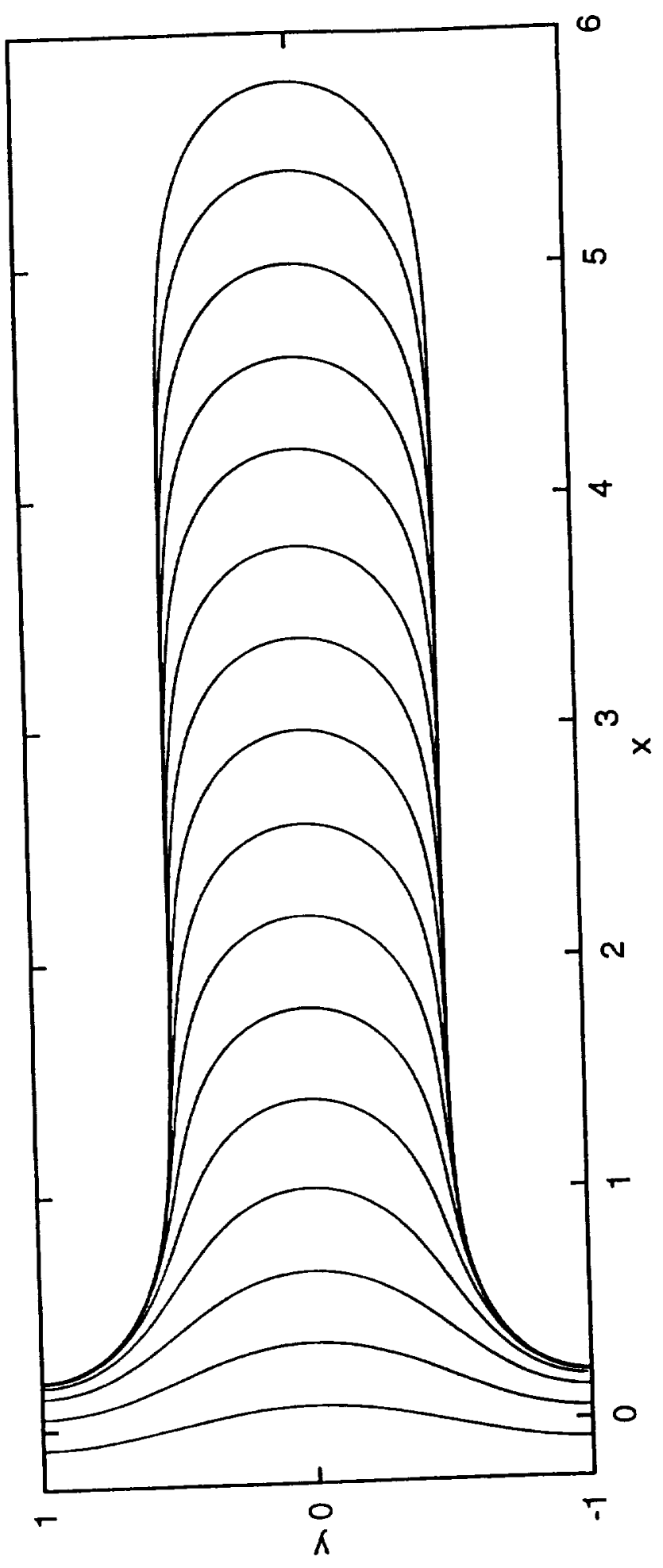

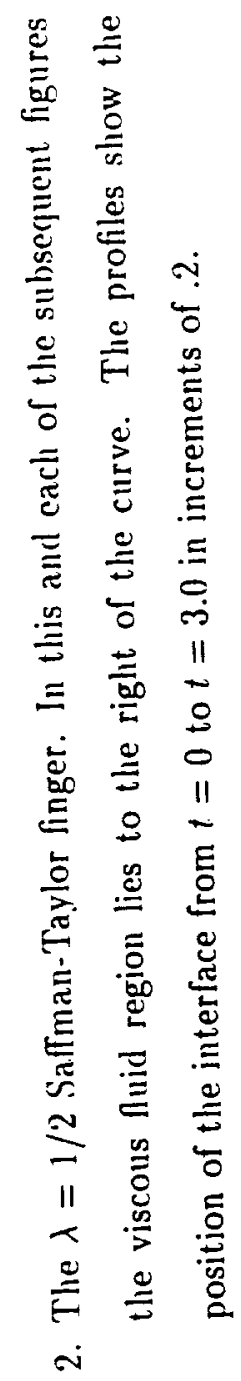




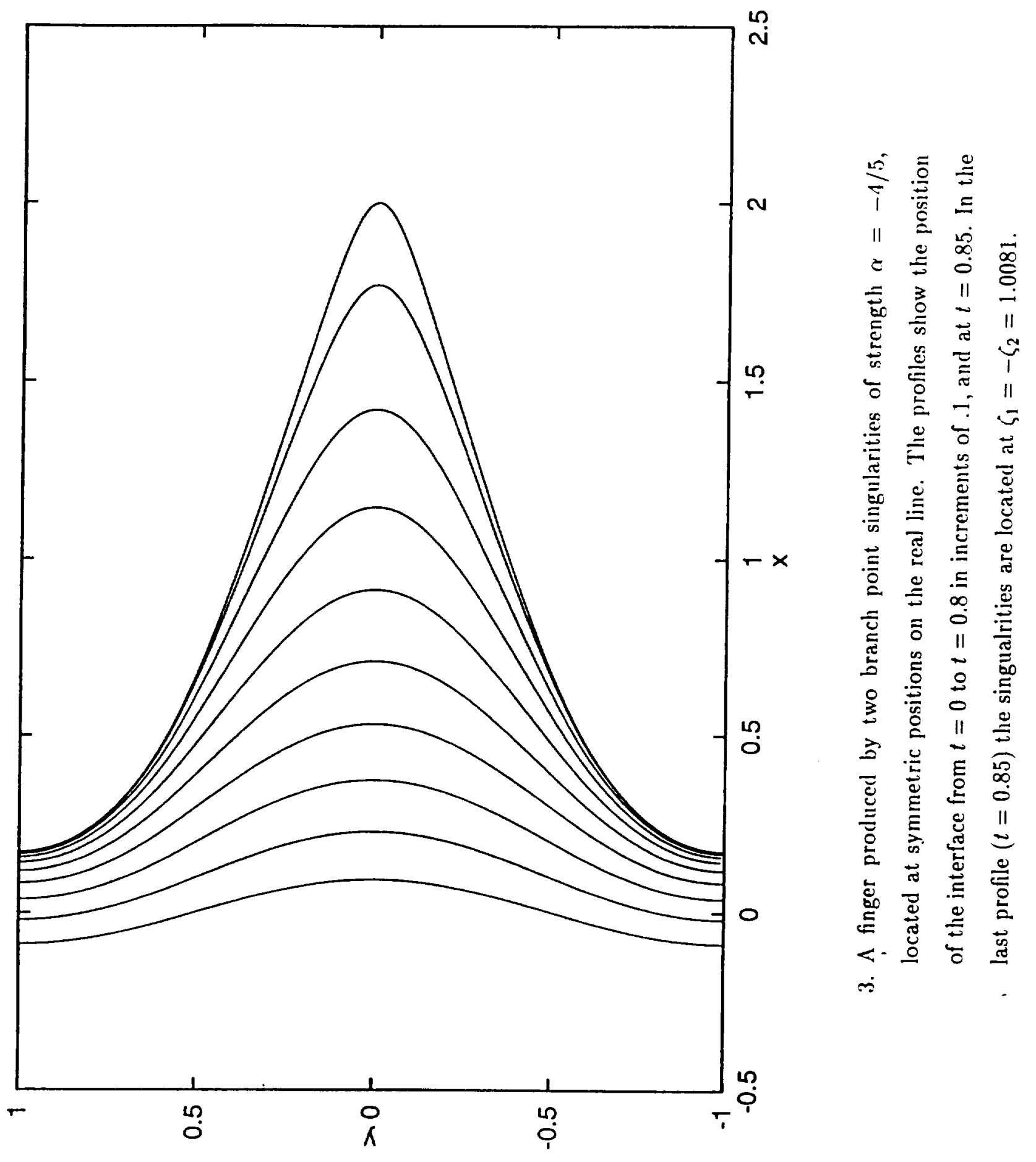




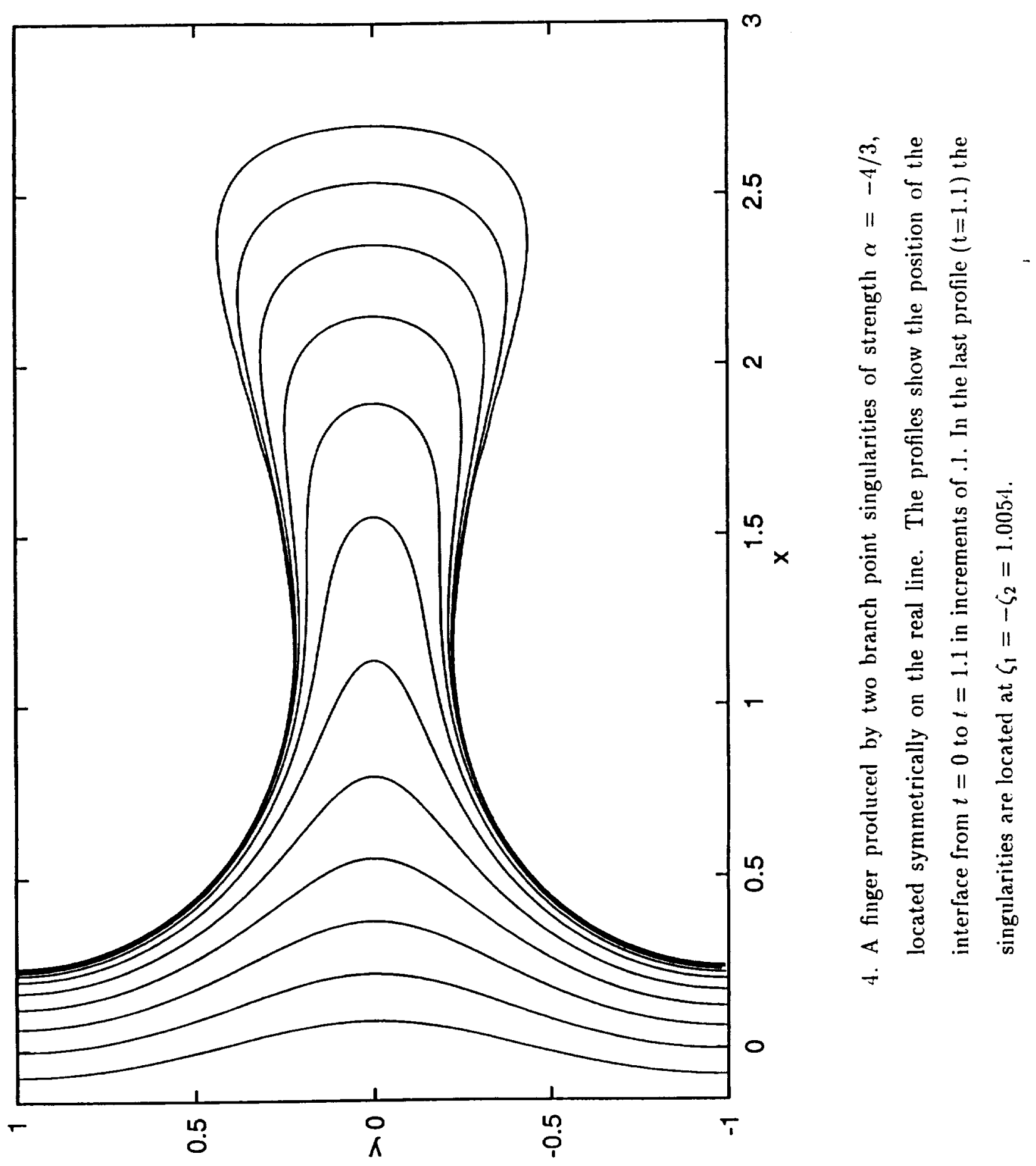




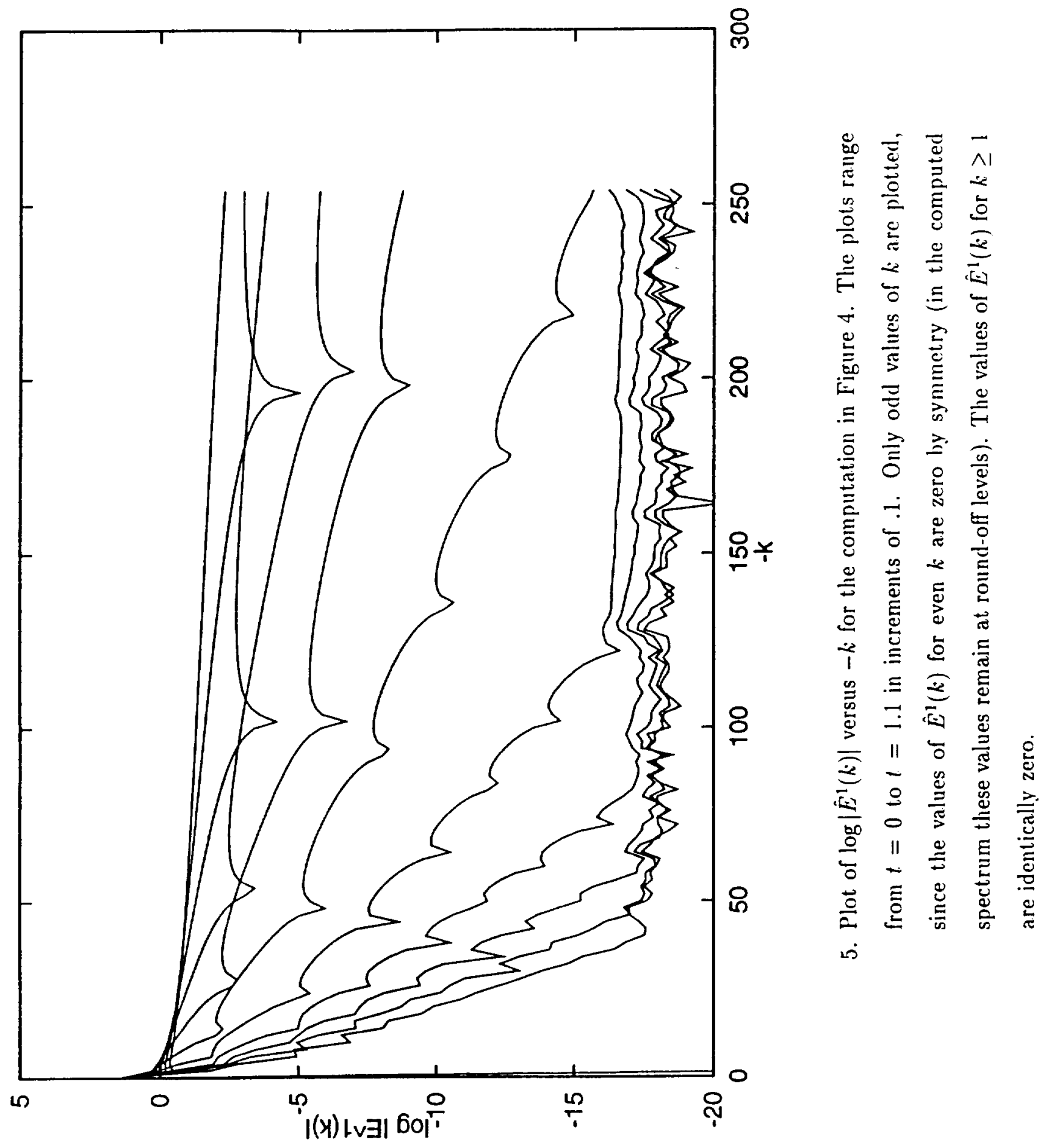




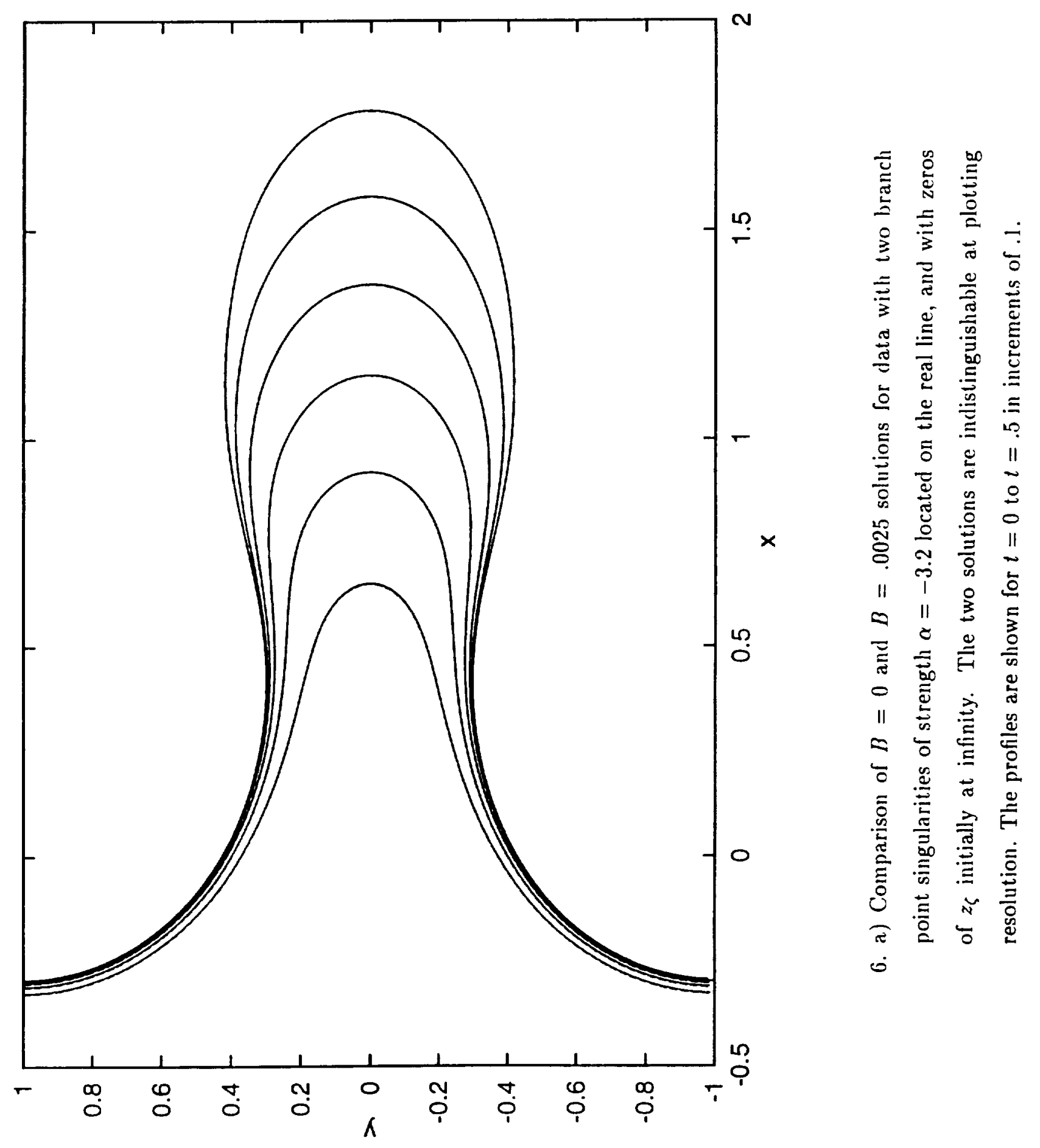




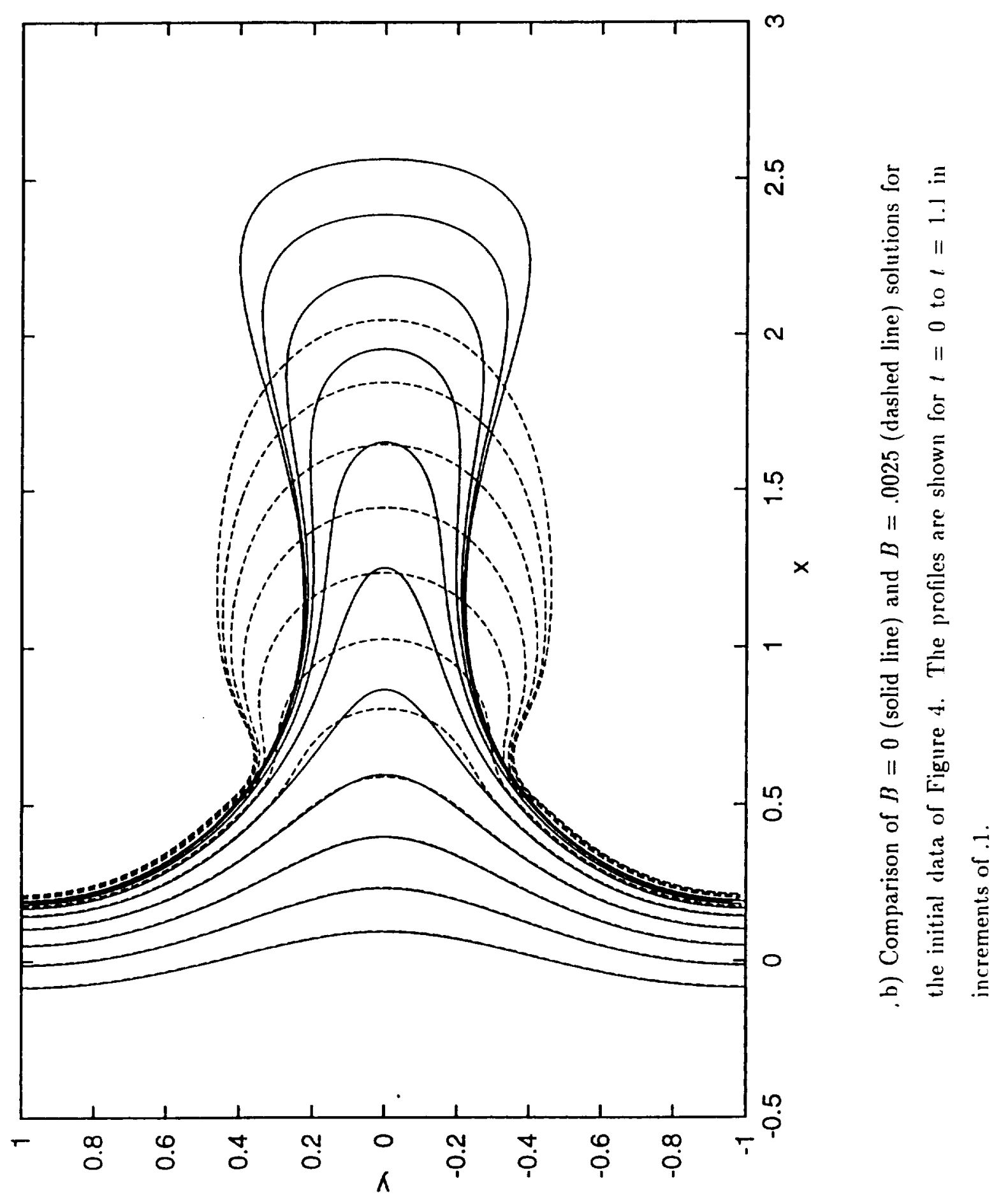




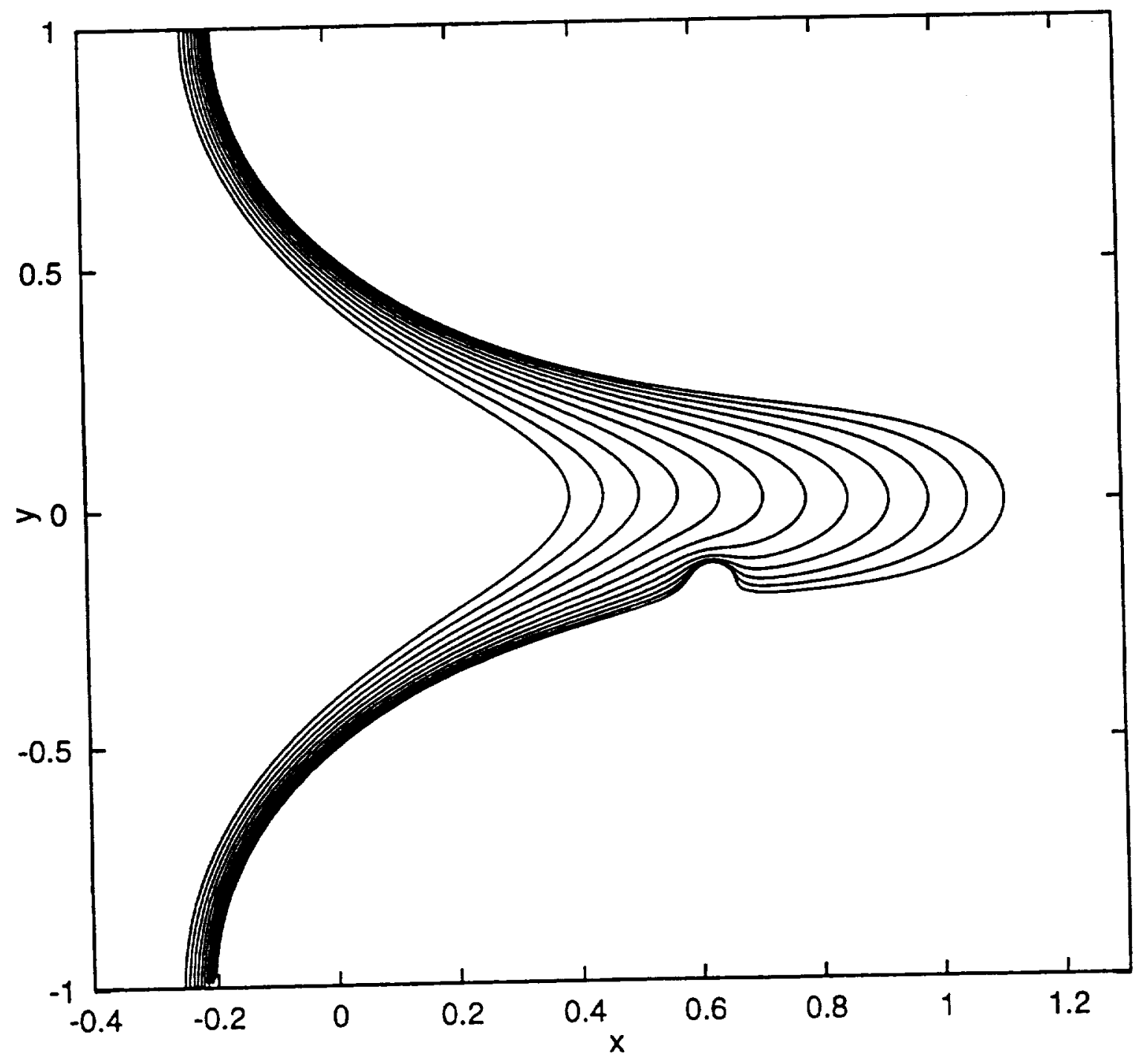

7. a) An advancing finger with a side perturbation. The plots range from $t=0$ to $t=0.22$ in increments of .02 . 


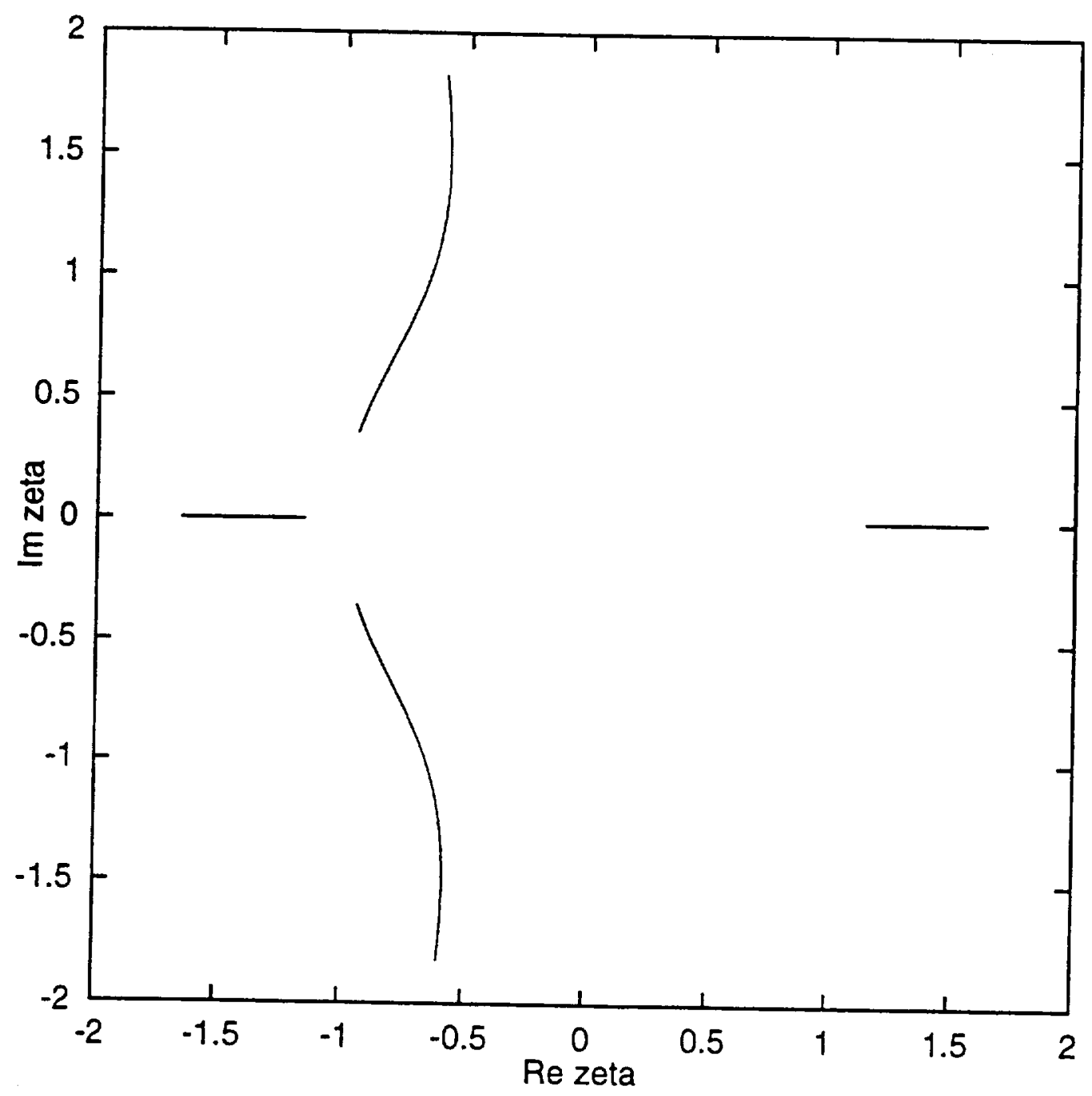

b) The singularity trajectories corresponding to $6(\mathrm{a})$. After reaching the unit circle, the (upper) complex singularity $\zeta_{3}$ moves in a counterclockwise direction. In the last profile $(l=.22)$, the singularities are located at $\zeta_{1}=1.1522, \zeta_{2}=$ -1.1542 , and $\zeta_{3}=(-.9381, .3546)$. 


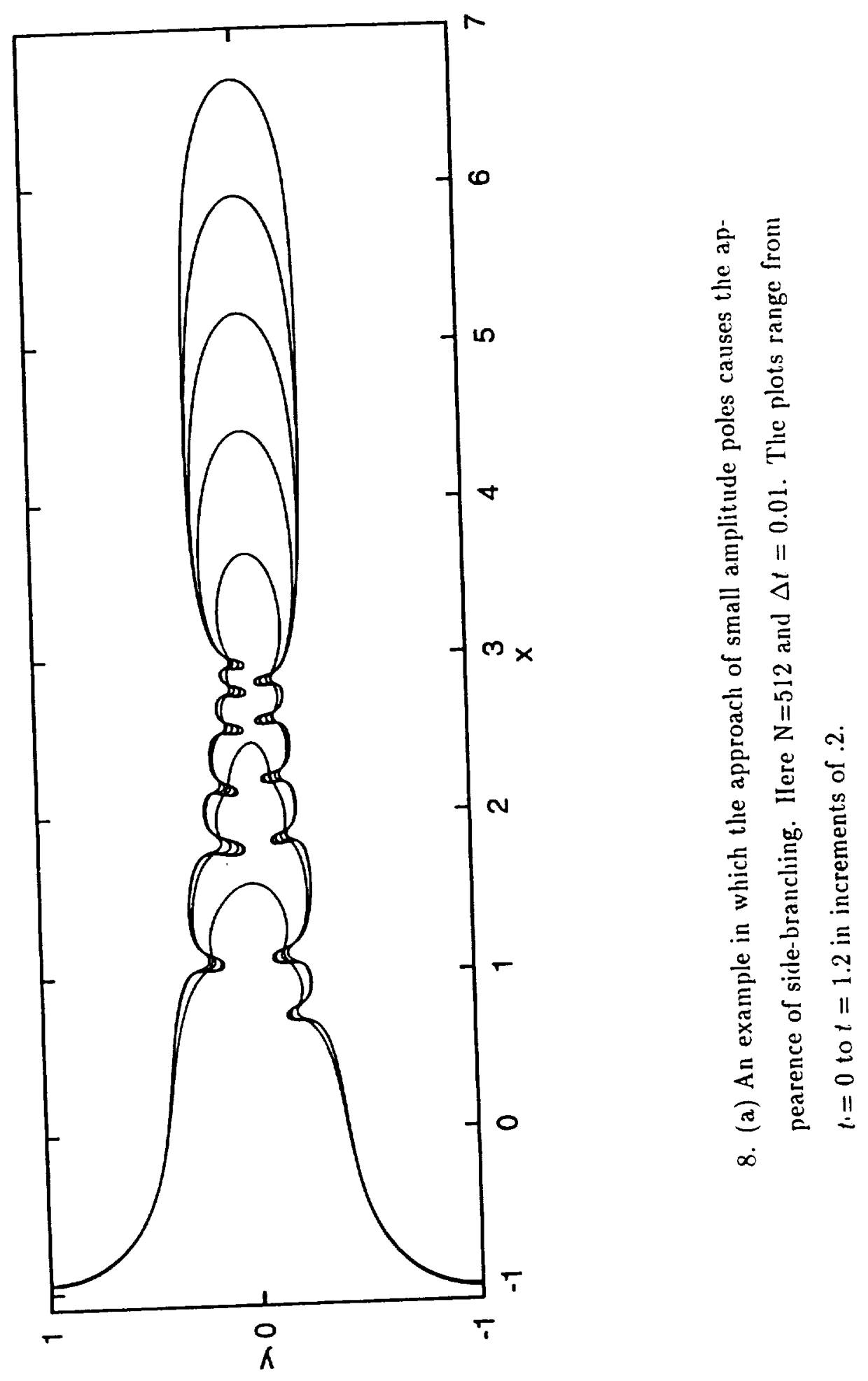




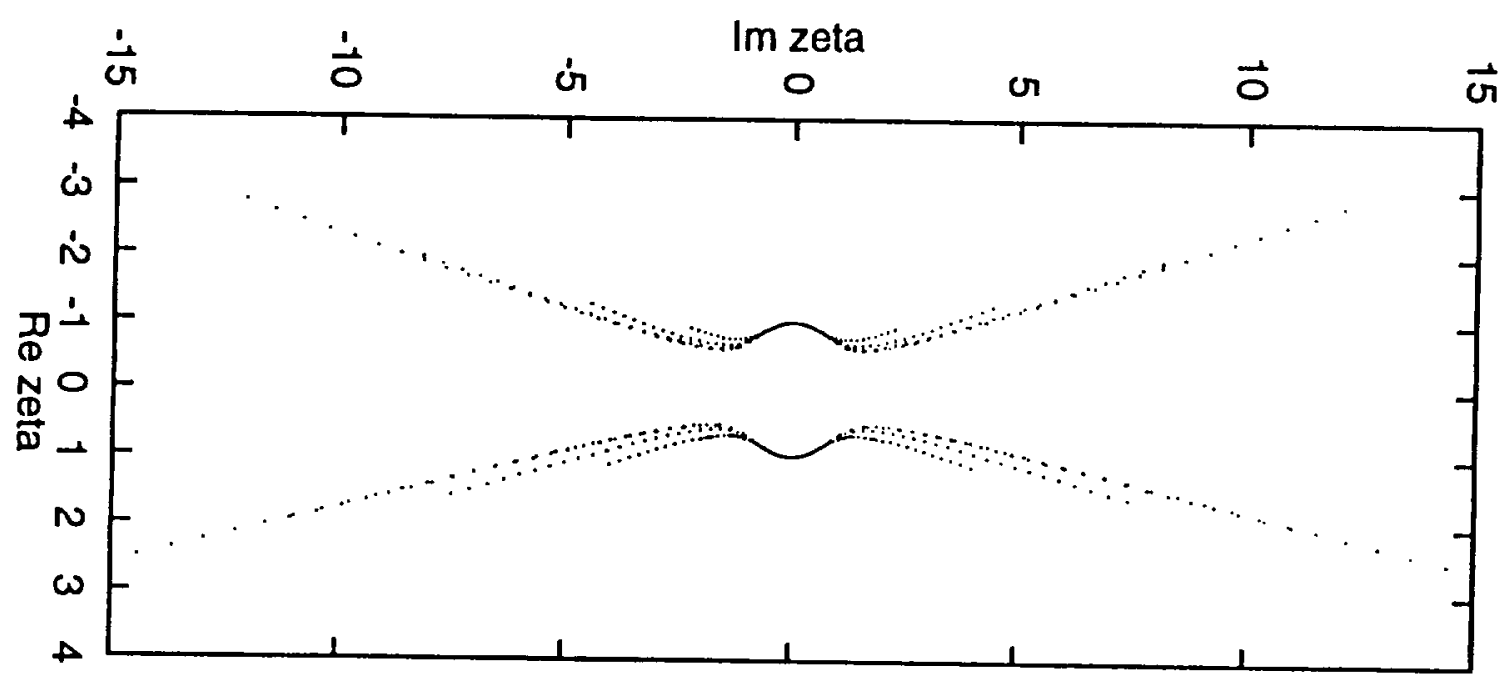

(b) The singularities trajectories corresponding to 7(a). 


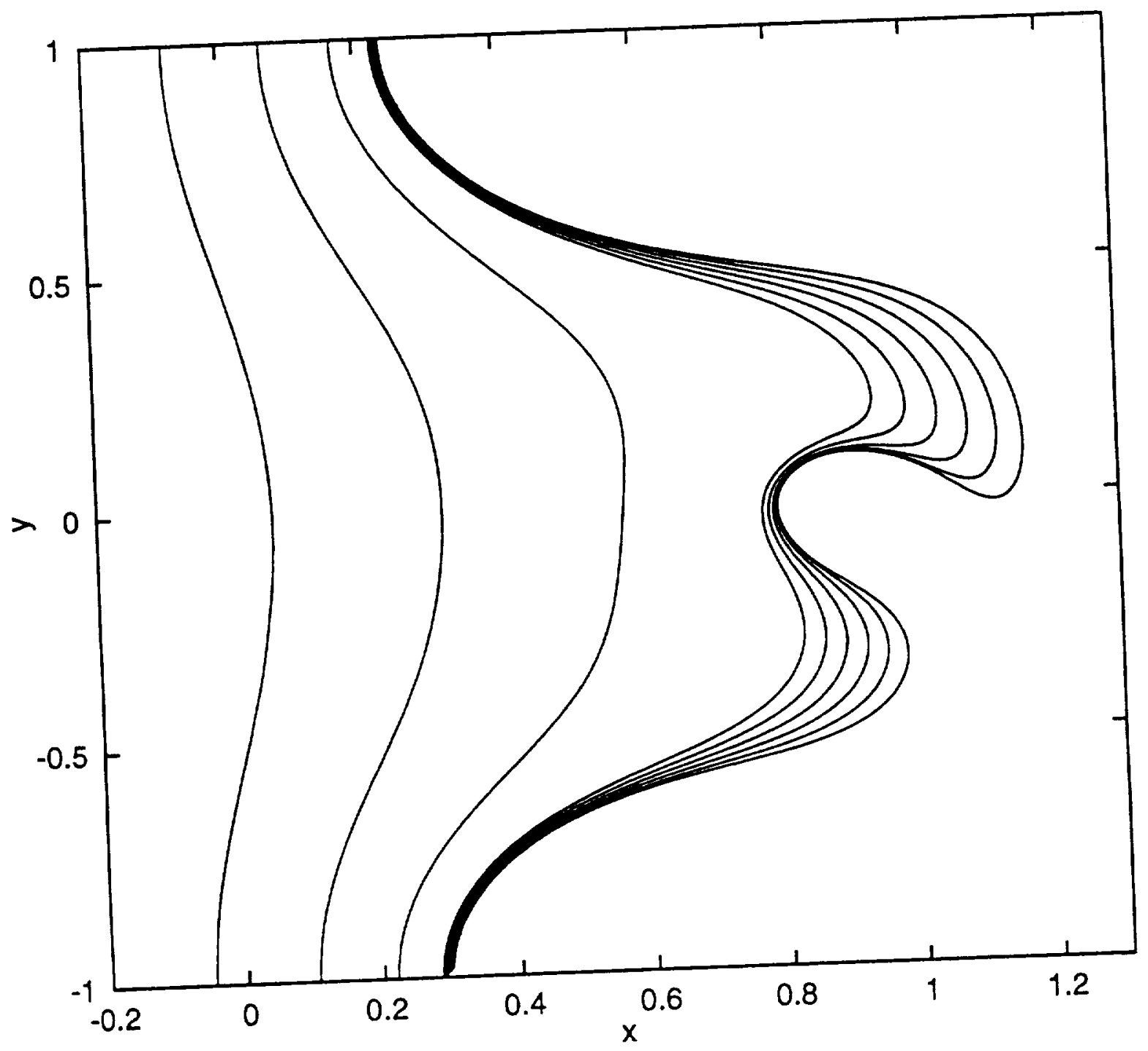

9. a) An illustration of tip splitting followed by finger formation and competition, due to $\alpha=-4 / 3$ branch point singularities. The plots range from from $t=0$ to $t=0.6 \mathrm{in}$ increments of .2 , and from $t=0.6$ to $t=0.7 \mathrm{in} \mathrm{increments} \mathrm{of} .02$. 


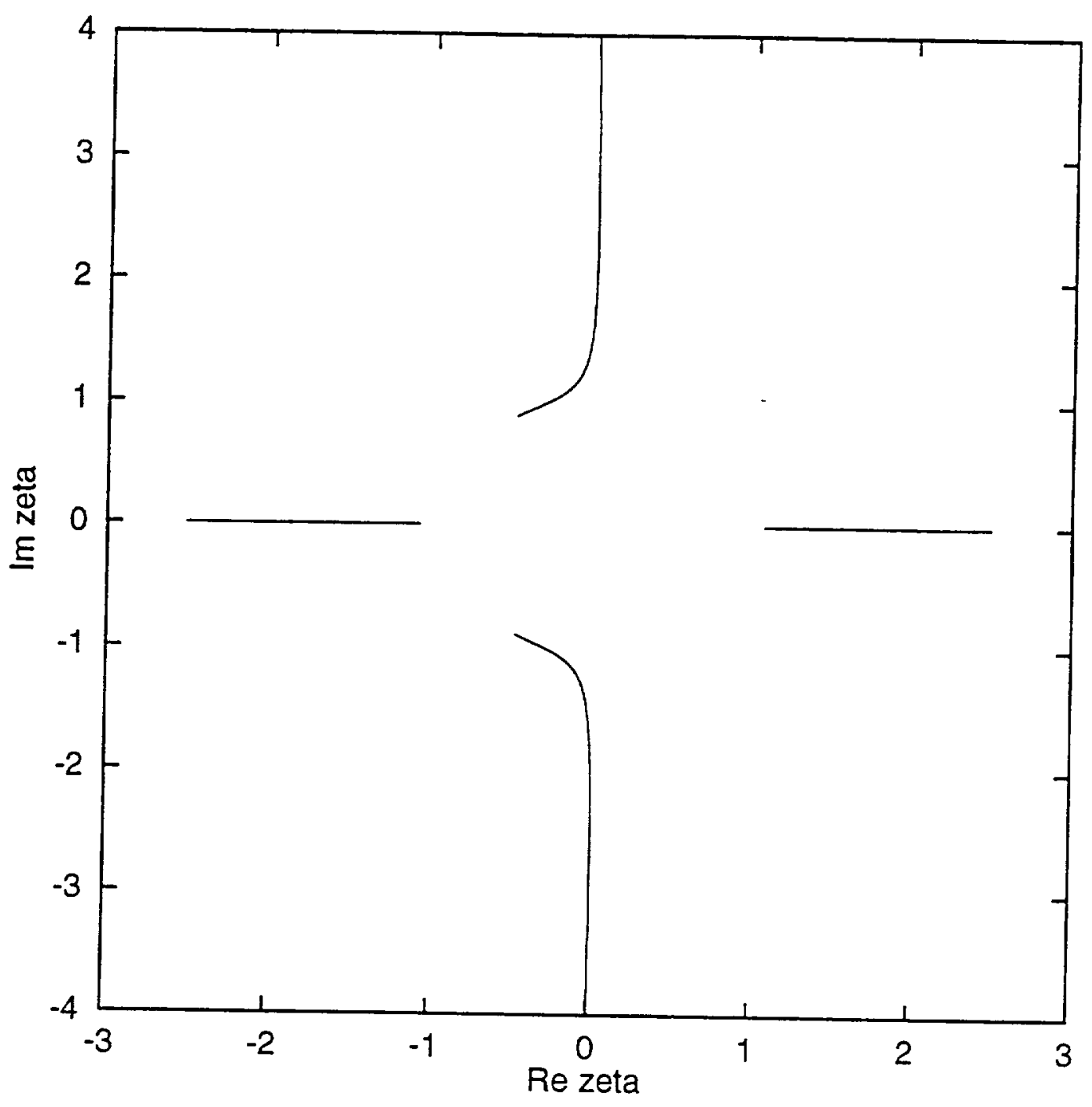

b) The singularity trajectories for the finger competition shown in $8(a)$. The motion of the singularities is towards $\zeta=-1$. 


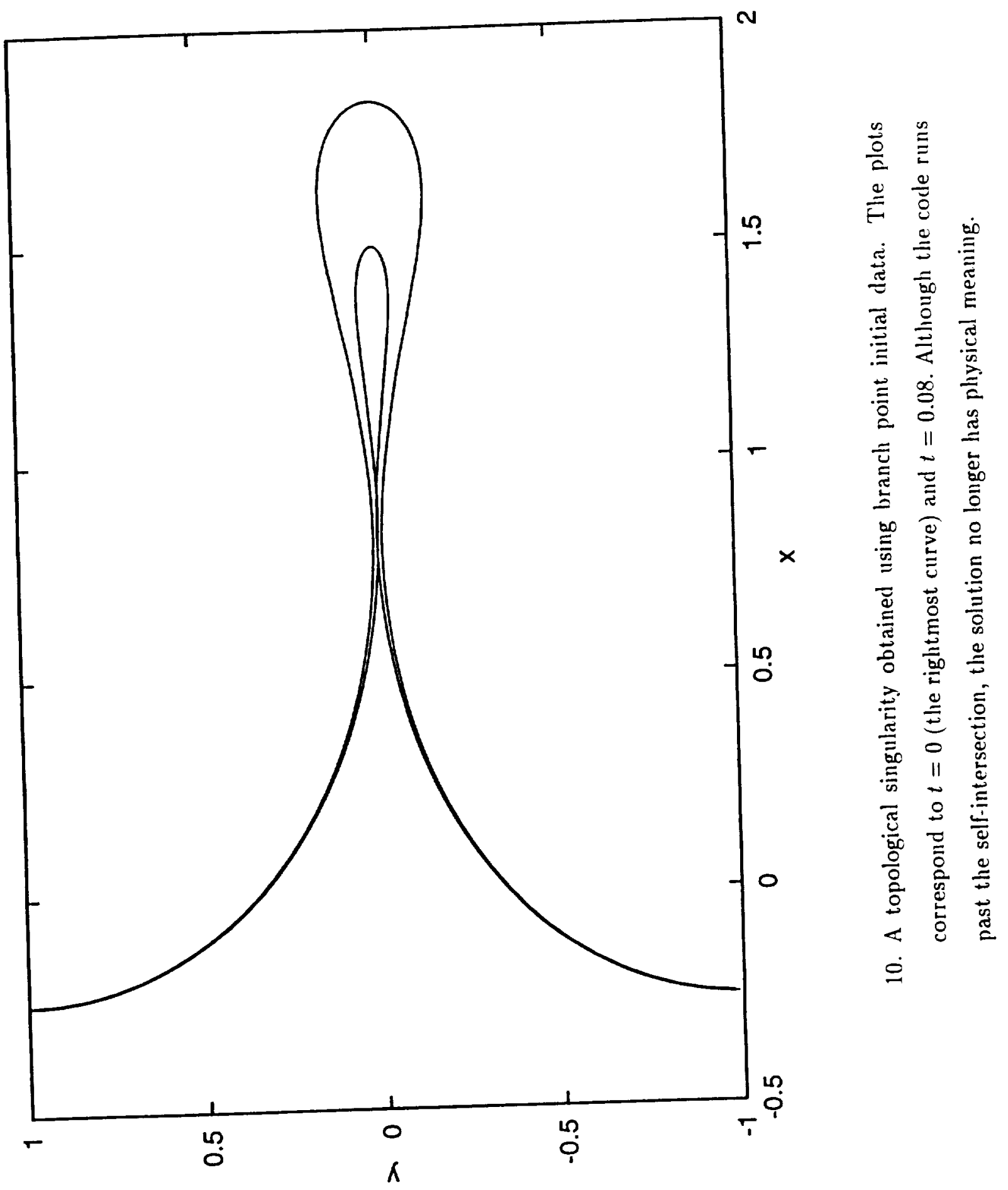





\begin{tabular}{|c|c|c|c|}
\hline \multicolumn{3}{|c|}{ REPORT DOCUMENTATION PAGE } & $\begin{array}{l}\text { Form Approved } \\
O M B \text { No } 0704-0188\end{array}$ \\
\hline \multicolumn{4}{|c|}{ 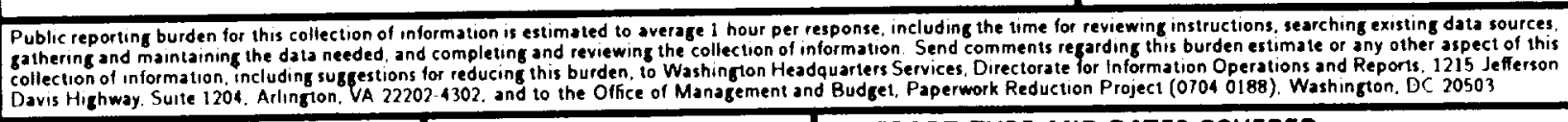 } \\
\hline 1. AGENCY USE ONLY(Leave blank) & $\begin{array}{l}\text { 2. REPORT DATE } \\
\text { February } 1995\end{array}$ & \multicolumn{2}{|c|}{$\begin{array}{l}\text { 3. REPORT TYPE AND DATES COVERED } \\
\text { Contractor Report }\end{array}$} \\
\hline \multicolumn{3}{|c|}{$\begin{array}{l}\text { 4. TITLE AND SUBTITLE } \\
\text { A WELL-POSED NUMERICAL METHOD TO TRACK ISO- } \\
\text { LATED CONFORMAL MAP SINGULARITIES IN HELE-SHAW } \\
\text { FLOW }\end{array}$} & \multirow[t]{2}{*}{$\begin{array}{l}\text { 5. FUNDING NUMBERS } \\
\text { C NAS1-19480 } \\
\text { WU } 505-90-52-01\end{array}$} \\
\hline \multicolumn{3}{|c|}{$\begin{array}{l}\text { 6. AUTHOR(S) } \\
\text { Gregory Baker } \\
\text { Michael Siegel } \\
\text { Saleh Tanveer }\end{array}$} & \\
\hline \multicolumn{3}{|c|}{$\begin{array}{l}\text { 7. PERFORMING ORGANIZATION NAME(S) ANO ADDRESS(ES) } \\
\text { Institute for Computer Applications in Science } \\
\text { and Engineering } \\
\text { Mail Stop 132C, NASA Langley Research Center } \\
\text { Hampton, VA 23681-0001 }\end{array}$} & $\begin{array}{l}\text { 8. PERFORMING ORGANIZATION } \\
\text { REPORT NUMBER } \\
\text { ICASE Report No. } 95-7\end{array}$ \\
\hline \multicolumn{3}{|c|}{$\begin{array}{l}\text { 9. SPONSORING/MONITORING AGENCY NAME(S) AND ADDRESS(ES) } \\
\text { National Aeronautics and Space Administration } \\
\text { Langley Research Center } \\
\text { Hampton, VA } 23681-0001\end{array}$} & $\begin{array}{l}\text { 10. SPONSORING/MONITORING } \\
\text { AGENCY REPORT NUMBER } \\
\text { NASA CR-195035 } \\
\text { ICASE Report No. } 95-7\end{array}$ \\
\hline \multicolumn{4}{|c|}{$\begin{array}{l}\text { 11. SUPPLEMENTARY NOTES } \\
\text { Langley Technical Monitor: Dennis M. Bushnell } \\
\text { Final Report } \\
\text { Submitted to Journal of Computational Physics }\end{array}$} \\
\hline \multicolumn{2}{|c|}{$\begin{array}{l}\text { 12a. DISTRIBUTION/AVAILABILITY STATEMENT } \\
\text { Unclassified-Unlimited } \\
\text { Subject Category } 64\end{array}$} & & 12b. OISTRIBUTION CODE \\
\hline \multicolumn{4}{|c|}{$\begin{array}{l}\text { 13. ABSTRACT (Maximum } 200 \text { mords) } \\
\text { We present a new numerical method for calculating an evolving 2-D Hele-Shaw interface when surface tension effects } \\
\text { are neglected. In the case where the flow is directed from the less viscous fluid into the more viscous fluid, the motion } \\
\text { of the interface is ill-posed; small deviations in the initial condition will produce significant changes in the ensuing } \\
\text { motion. This situation is disastrous for numerical computation, as small round-off errors can quickly lead to large } \\
\text { inaccuracies in the computed solution. Our method of computation is most easily formulated using a conformal map } \\
\text { from the fluid domain into a unit disk. The method relies on analytically continuing the initial data and equations } \\
\text { of motion into the region exterior to the disk, where the evolution problem becomes well-posed. The equations are } \\
\text { then numerically solved in the extended domain. The presence of singularities in the conformal map outside of the } \\
\text { disk int roduces specific structures along the fluid interface. Our method can explicitly track the location of isolated } \\
\text { pole and branch point singularities, allowing us to draw connections between the development of interfacial patterns } \\
\text { and the motion of singularities as they approach the unit disk. In particular, we are able to relate physical features } \\
\text { such as finger shape, side-branch formation, and competition between fingers to the nature and location of the } \\
\text { singularities. The usefulness of this method in studying the formation of topological singularities (self-intersections } \\
\text { of the interface) is also pointed out. }\end{array}$} \\
\hline \multirow{2}{*}{\multicolumn{3}{|c|}{$\begin{array}{l}\text { 14. SUBJECT TERMS } \\
\text { Hele-Shaw instability; ill-posed; singularities; side-branching; finger competition }\end{array}$}} & $\begin{array}{l}\text { 15. NUMBER OF PAGES } \\
50\end{array}$ \\
\hline & & & $\begin{array}{r}\text { 16. PRICE CODE } \\
\mathrm{A} 03 \\
\end{array}$ \\
\hline $\begin{array}{l}\text { 17. SECURITY CLASSIFICATION } \\
\text { OF REPORT } \\
\text { Cinclassified }\end{array}$ & $\begin{array}{l}\text { 18. SECURITY CLASSIFICATION } \\
\text { OF THIS PAGE } \\
\text { Unclassified }\end{array}$ & $\begin{array}{l}\text { 19. SECURITY CLASSIFICATION } \\
\text { OF ABSTRACT }\end{array}$ & $\begin{array}{l}\text { 20. LIMITATION } \\
\text { OF ABSTRACT }\end{array}$ \\
\hline NSN 7540-01-260-5500 & & & $\begin{array}{l}\text { Standard form } 298(\text { Rev. } 2.89) \\
\text { Prescribed by ANSI S1d } 239.18 \\
298.102\end{array}$ \\
\hline
\end{tabular}

